\title{
Article
}

\section{Box/peanut-shaped bulges in action space}

Debattista, Victor P, Liddicott, David John, Khachaturyants, Tigran and Beraldo E silva, Leandro

Available at http://clok.uclan.ac.uk/34796/

Debattista, Victor P ORCID: 0000-0001-7902-0116, Liddicott, David John, Khachaturyants, Tigran and Beraldo E silva, Leandro ORCID: 0000-0002-07401507 (2020) Box/peanut-shaped bulges in action space. Monthly Notices of the Royal Astronomical Society, 498 (3). pp. 3334-3350. ISSN 0035-8711

It is advisable to refer to the publisher's version if you intend to cite from the work. http://dx.doi.org/10.1093/mnras/staa2568

For more information about UCLan's research in this area go to http://www.uclan.ac.uk/researchgroups/ and search for <name of research Group>.

For information about Research generally at UCLan please go to http://www.uclan.ac.uk/research/

All outputs in CLoK are protected by Intellectual Property Rights law, including Copyright law. Copyright, IPR and Moral Rights for the works on this site are retained by the individual authors and/or other copyright owners. Terms and conditions for use of this material are defined in the policies page.

\section{CLoK}

Central Lancashire online Knowledge www.clok.uclan.ac.uk

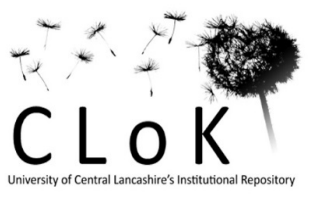




\title{
Box/peanut-shaped bulges in action space
}

\author{
Victor P. Debattista ${ }^{1 \star}$, David J. Liddicott ${ }^{1}$, Tigran Khachaturyants ${ }^{1}$, \\ Leandro Beraldo e Silva ${ }^{1}$ \\ 1 Jeremiah Horrocks Institute, University of Central Lancashire, Preston, PR1 2HE, UK
}

Draft version on 23 August 2020

\begin{abstract}
We introduce the study of box/peanut $(\mathrm{B} / \mathrm{P})$ bulges in the action space of the initial axisymmetric system. We explore where populations with different actions end up once a bar forms and a $\mathrm{B} / \mathrm{P}$ bulge develops. We find that the density bimodality due to the $\mathrm{B} / \mathrm{P}$ bulge (the $\mathrm{X}$-shape) is better traced by populations with low radial, $\mathrm{J}_{\mathrm{R}, 0}$, or vertical, $\mathrm{J}_{z, 0}$, actions, or high azimuthal action, $\mathrm{J}_{\phi, 0}$. Generally populations separated by $\mathrm{J}_{\mathrm{R}, 0}$ have a greater variation in bar strength and vertical heating than those separated by $J_{z, 0}$. While the bar substantially weakens the initial vertical gradient of $J_{z, 0}$, it also drives a strikingly monotonic vertical profile of $J_{\mathrm{R}, 0}$. We then use these results to guide us in assigning metallicity to star particles in a pure $N$-body model. Because stellar metallicity in unbarred galaxies depends on age as well as radial and vertical positions, the initial actions are particularly well suited for assigning metallicities. We argue that assigning metallicities based on single actions, or on positions, results in metallicity distributions inconsistent with those observed in real galaxies. We therefore use all three actions to assign metallicity to an $N$-body model by comparing with the actions of a star-forming, unbarred simulation. The resulting metallicity distribution is pinched on the vertical axis, has a realistic vertical gradient and has a stronger $\mathrm{X}$-shape in metal-rich populations, as found in real galaxies.
\end{abstract}

Key words: Galaxy: abundances - Galaxy: bulge - Galaxy: evolution — Galaxy: stellar content — Galaxy: structure — galaxies: bulges

\section{INTRODUCTION}

Among the integrals of motion that have been used to study the Milky Way (MW), actions have found particular favour in part because they can be computed directly from stellar orbits (Binney \& Tremaine 2008), or approximated from instantaneous positions and velocities under the assumption that the potential is locally of Stäckel form (Binney 2012). The adiabatic growth of the MW's mass conserves the actions and stars should retain some memory of the state of the MW when the stars were born. However various resonant processes alter the actions. Scattering, such as by giant molecular clouds, drives a slow diffusion in action space (Binney \& Lacey 1988) which manifests as the slow heating of stellar populations (Spitzer \& Schwarzschild 1951, 1953; Wielen 1977; Icke 1982; Lacey 1984; Villumsen 1985). Scattering can be viewed as the resonant response to collective modes excited by fluctuations (Nelson \& Tremaine 1999; Heyvaerts 2010; Sellwood 2013, 2015; Heyvaerts et al. 2017; Fouvry \& Bar-Or 2018). Actions are changed more substan-

* E-mail: vpdebattista@gmail.com tially at resonances of global perturbations, such as the inner and outer Lindblad resonances (Barbanis \& Woltjer 1967; Lynden-Bell \& Kalnajs 1972; Carlberg \& Sellwood 1985), and the corotation resonance (Sellwood \& Binney 2002; Roškar et al. 2012; Daniel \& Wyse 2015) of bars and spirals. The resulting diffusion in action space can be anisotropic; for instance during the migration driven by the spiral corotation resonance, the radial action is largely conserved, while the azimuthal action changes dramatically (Sellwood \& Binney 2002; Roškar et al. 2012). The vertical action is conserved on average during corotation migration, but not in detail (on a star by star basis), because of vertical resonances (Solway et al. 2012; Vera-Ciro \& D'Onghia 2016).

During dynamical instabilities the potential can change rapidly. In particular, the bar (Toomre 1981; Sellwood 1981) and the buckling instabilities (Raha et al. 1991) are generally violent and unlikely to conserve the actions. Notwithstanding this, the initial actions determine how the stars react to the perturbation and thus help in understanding the final distributions of stars within a $\mathrm{B} / \mathrm{P}$ bulge once it forms. Debattista et al. (2017) showed that the initial radial random motions play a large role in determining the 
morphology of different populations within the $\mathrm{B} / \mathrm{P}$ bulge. The formation of $\mathrm{B} / \mathrm{P}$ bulges has a long history of study using numerical simulations (Combes \& Sanders 1981; Combes et al. 1990; Pfenniger \& Friedli 1991; Raha et al. 1991; Merritt \& Sellwood 1994; Berentzen et al. 1998; O'Neill \& Dubinski 2003; Martinez-Valpuesta \& Shlosman 2004; Debattista et al. 2004, 2005, 2006; Athanassoula 2005; Bureau \& Athanassoula 2005; Martinez-Valpuesta et al. 2006; Shen et al. 2010; Martinez-Valpuesta \& Gerhard 2011, 2013; Saha \& Gerhard 2013; Saha et al. 2013; Li \& Shen 2012, 2015; Di Matteo et al. 2014, 2015; Di Matteo 2016; Fragkoudi et al. 2017, 2018; Smirnov \& Sotnikova 2019; Łokas 2019; Collier 2020). In this paper we introduce the study of $B / P$ bulges in the space of the initial actions. This leads to new insights into how $\mathrm{B} / \mathrm{P}$ bulges form and suggests new avenues of study.

A further motivation for exploring how initial actions map into a $\mathrm{B} / \mathrm{P}$ bulge once one forms is that we can use the actions to assign stellar populations to the bulge. Compared with pure $N$-body simulations, simulations that include the physics of gas and star formation remain expensive and do not permit rapid explorations of a large parameter space, which are needed to test ideas of bulge formation. With the European Space Agency's Gaia mission now in its second data release and the third data release imminent, such models are vital for a detailed understanding of the formation of the MW's bulge. Therefore a prescription for assigning stellar population properties (ages, metallicities, abundances, etc.) to the star particles in pure $N$-body simulations would be very valuable. This action-based metallicity prescription is different from past prescriptions based solely on position and membership in the thin or thick disc (e.g. Bekki \& Tsujimoto 2011; Martinez-Valpuesta \& Gerhard 2013; Di Matteo 2016) because stars at any particular location can be a mix of very different metallicities (Hayden et al. 2015).

This paper is organised as follows. Section 2 presents the simulation suite used in this paper. Section 3 presents the evolution of stellar populations tagged by actions in a fiducial simulation while Section 4 generalises these results to the entire simulation suite. In Section 5 we consider prescriptions for assigning metallicity to stars based on their initial actions. We discuss our results and present our conclusions in Section 6. The deconstruction of barred models by actions has never been presented before. Therefore in the online version of the paper the appendices present figures for a standard analysis of all the other simulations.

\section{SIMULATIONS}

We use a suite of pure $N$-body simulations to track stars based on their actions. The models were set up using GALACTICS (Widrow et al. 2008; Widrow \& Dubinski 2005; Kuijken \& Dubinski 1995); they are comprised of one or two discs in a truncated Navarro-Frenk-White (NFW) dark matter halo (Navarro et al. 1996):

$$
\rho(r)=\frac{2^{2-\gamma} \sigma_{h}^{2}}{4 \pi a_{h}^{2}} \frac{C(r)}{\left(r / a_{h}\right)^{\gamma}\left(1+r / a_{h}\right)^{3-\gamma}},
$$

\begin{tabular}{cccccc}
\hline Model & $\begin{array}{c}\sigma_{R 0} \\
{\left[\mathrm{~km} \mathrm{~s}^{-1}\right]}\end{array}$ & $\begin{array}{c}z_{\mathrm{d}} \\
{[\mathrm{pc}]}\end{array}$ & $r_{\phi, R}$ & $r_{\phi, z}$ & $r_{R, z}$ \\
\hline 1 & 90 & 300 & -0.39 & -0.34 & 0.18 \\
2 & 128 & 300 & -0.41 & -0.35 & 0.19 \\
3 & 165 & 300 & -0.41 & -0.36 & 0.18 \\
4 & 128 & 150 & -0.40 & -0.37 & 0.20 \\
5 & 128 & 600 & -0.42 & -0.32 & 0.18 \\
T1 & $60+90$ & $100+400$ & -0.18 & -0.25 & 0.24 \\
T5 & $100+140$ & $300+900$ & -0.26 & -0.25 & 0.19 \\
HD1 & 45 & 300 & -0.38 & -0.32 & 0.16 \\
HD2 & 60 & 300 & -0.39 & -0.33 & 0.18 \\
\hline
\end{tabular}

Table 1. The models used in this paper. $\sigma_{R 0}$ is the disc's central radial velocity dispersion(s), $z_{\mathrm{d}}$ is the disc scale height(s), and $r_{i, j}$ is the Pearson $r$ for the correlation between actions $J_{i, 0}$ and $\mathrm{J}_{\mathrm{j}, 0}$.

(Widrow et al. 2008), where the cutoff function $C(r)$ smoothly truncates the model at a finite radius:

$$
C(r)=\frac{1}{2} \operatorname{erfc}\left(\frac{r-r_{h}}{\sqrt{2} \delta r_{h}}\right) \text {. }
$$

For all models we set $\sigma_{h}=400 \mathrm{~km} \mathrm{~s}^{-1}, a_{h}=16.7 \mathrm{kpc}, \gamma=$ $0.873, r_{h}=100 \mathrm{kpc}$, and $\delta r_{h}=25 \mathrm{kpc}$.

The discs are exponential with an isothermal vertical profile:

$$
\Sigma(R, z)=\Sigma_{0} \exp \left(-R / R_{\mathrm{d}}\right) \operatorname{sech}^{2}\left(z / z_{\mathrm{d}}\right)
$$

where $R_{\mathrm{d}}$ and $z_{\mathrm{d}}$ are the disc's scale-length and scale-height, respectively. For models $1-5$ we set $M_{\mathrm{d}}=2 \pi \Sigma_{0} R_{\mathrm{d}}{ }^{2}=$ $5.2 \times 10^{10} \mathrm{M}_{\odot}$, and $R_{\mathrm{d}}=2.4 \mathrm{kpc}$. Figure 1 of Debattista et al. (2017) shows the rotation curve of these systems, which reyeals that the galaxy is baryon-dominated out to $\sim 10 \mathrm{kpc}$. In this way the models resemble the MW (Cole \& Binney 2017). The kinematics of the discs are set such that the radial velocity dispersion, $\sigma_{R}$, decreases exponentially

$$
\sigma_{R}^{2}(R)=\sigma_{R 0}^{2} \exp \left(-R / R_{\sigma}\right) .
$$

We set $R_{\sigma}=2.5 \mathrm{kpc}$. In models $1-5$ we vary the radial and vertical dispersions as listed in Table 1. Our fiducial model is model 2 , while models 1 and 3 bracket it in radial random motions, and 4 and 5 bracket it in vertical random motions (i.e. thickness). With these choices the minimum of the Toomre- $Q$ profiles ranges from 0.8 to 1.5 . We use $6 \times 10^{6}$ particles in the disc and $4 \times 10^{6}$ particles in the halo. Thus disc particles have a mass $\simeq 9 \times 10^{3} \mathrm{M}_{\odot}$, while halo particles have mass $\simeq 1.7 \times 10^{5} \mathrm{M}_{\odot}$.

Our simulation suite also includes two thin+thick disc models, T1 and T5, the former taken from Debattista et al. (2017). The two discs both have $R_{\mathrm{d}}=2.4 \mathrm{kpc}$, each of mass $2.6 \times 10^{10} \mathrm{M}_{\odot}$, so the total stellar mass is the same as in models 1-5. In model $\mathrm{T} 1$, the thin disc has $\left(z_{\mathrm{d}}, \sigma_{R 0}, R_{\sigma}\right)=\left(100 \mathrm{pc}, 60 \mathrm{~km} \mathrm{~s}^{-1}, 4.5 \mathrm{kpc}\right)$ while for the thick disc these values are $\left(400 \mathrm{pc}, 90 \mathrm{~km} \mathrm{~s}^{-1}, 2.5 \mathrm{kpc}\right)$. The evolution of model T1 is described in Section 3.2 of Debattista et al. (2017). In model T5, which has not been presented elsewhere, we set a thin disc with $\left(z_{\mathrm{d}}, \sigma_{R 0}, R_{\sigma}\right)=$ $\left(300 \mathrm{pc}, 100 \mathrm{~km} \mathrm{~s}^{-1}, 4.5 \mathrm{kpc}\right)$ while for the thick disc these parameters are $\left(900 \mathrm{pc}, 140 \mathrm{~km} \mathrm{~s}^{-1}, 3.5 \mathrm{kpc}\right)$. The dark matter halo of both models is the same as that in models 1-5.

We also include two dark matter-dominated systems, 


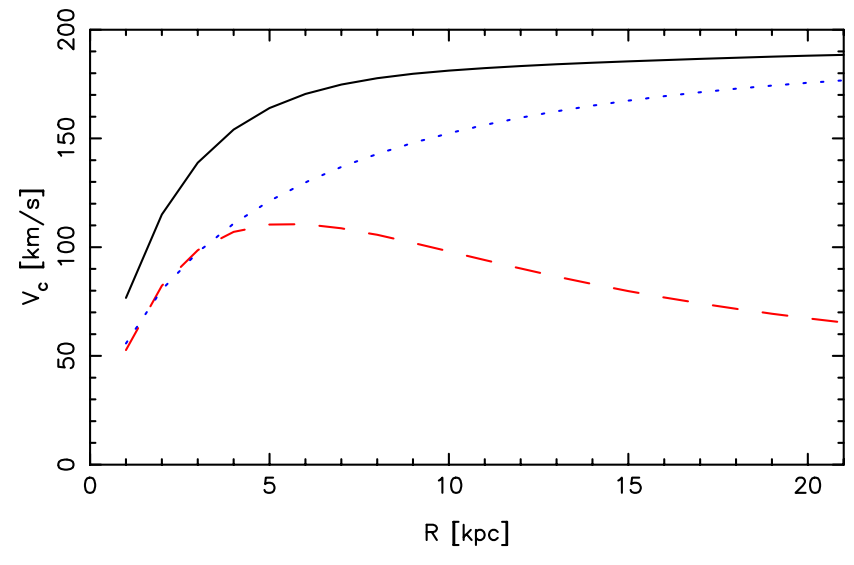

Figure 1. The rotation curve of the halo-dominated models, HD1 and HD2. The solid (black) line shows the total rotation curve while the dashed (red) and dotted (blue) lines show the disc and halo contributions, respectively.

models HD1 and HD2 ("halo dominated"), in which the dark matter and baryons contribute about equally to the inner rotation curve, as shown in Fig. 1. In these models we keep the same halo density profile as in the other models, and the same disc scale-length, $R_{\mathrm{d}}$, scale-height, $z_{\mathrm{d}}$, and dispersion scale-length, $R_{\sigma}$, as in the fiducial model. The only two differences are that we lower the mass of the disc to $35 \%$ of the fiducial model and lower the central velocity dispersion to have Toomre- $Q$ profiles similar to model 1 (HD1) and model 2 (HD2).

The models were evolved with PKDGRAV (Stadel 2001), using a particle softening of $\epsilon=50 \mathrm{pc}$ and $\epsilon=100 \mathrm{pc}$ for star and halo particles, respectively, in all the simulations. The base timestep is $\Delta t=5 \mathrm{Myr}$, and is refined such that each particle's timestep satisfies $\delta t=\Delta t / 2^{n}<\eta \sqrt{\epsilon / a_{g}}$, where $a_{g}$ is the acceleration at the particle's current position. We use $\eta=0.2$ and set the opening angle of the tree code gravity calculation $\theta=0.7$. We evolve the models for $5 \mathrm{Gyr}$, except for models HD1 and HD2, which we evolve for 10 Gyr since their bars take longer to form. During this time a bar forms and buckles, resulting in a $\mathrm{B} / \mathrm{P}$ bulge. At the end of the simulations we align the bars along the $x$-axis to facilitate our analysis and inter-comparison.

Fig. 2 presents the evolution of the bar and the buckling global amplitudes of the full simulation suite. We define these amplitudes as:

and

$$
A_{\mathrm{bar}}=\left|\frac{\sum_{k} m_{k} e^{2 i \phi_{k}}}{\sum_{k} m_{k}}\right|,
$$

where $m_{k}, z_{k}$ and $\phi_{k}$ are the mass, vertical position and azimuthal angle of particle $k$ and the sums are over all star particles (e.g. Sellwood \& Athanassoula 1986; Debattista et al. 2006). The models exhibit a range of bar growth rates, and bar strengths. All bars weaken at buckling. By the end of the simulations most of the bars have started to grow again. The fiducial model 2 forms a bar at $t \sim 1 \mathrm{Gyr}$, and buckles at $t \sim 3$ Gyr. The cooler model 1 forms its bar faster and buckles at $t \sim 3.7$ Gyr. The hotter model 3 forms its bar later still, but still buckles at $t \sim 3.7 \mathrm{Gyr}$. The thinner (thicker) model 4 (5) forms earlier (later). Buckling in model 5 is the strongest of the entire simulation suite. The bar in model T1 forms quite rapidly and buckles quite mildly, whereas model T5 forms a bar somewhat later and undergoes a strong buckling. Model HD1 forms a bar at $\sim 4 \mathrm{Gyr}$, and buckles at 6 Gyr. After $t \sim 7$ Gyr $A_{\text {buck }}$ rises monotonically but this reflects a bend in the bar rather than a buckling. Finally model HD2 forms a bar at 7 Gyr and buckles at $t \sim 9.2$ Gyr.

Fig. 3 presents a view of model 2 at the start and end of the simulation. The main analysis presented will focus on this model. Plots similar to those for model 2 for the rest of the simulation suite are presented in the Appendices of the online edition.

\section{EVOLUTION IN INITIAL ACTION SPACE}

We use AGAMA (Vasiliev 2019) to measure the actions in the radial, vertical and azimuthal directions at $t=0$. We compute the potential of the disc and halo separately, assuming spherical symmetry for the halo and a flattened axisymmetric distribution for the disc. We have verified that the total potential computed this way reproduces the rotation curve. We then compute the actions assuming the local axisymmetric Stäckel fudge (Binney 2012).

We treat the actions computed at $t=0$ as tags for stellar particles at the end of the simulation. We stress that at the end of the simulations these are still the actions computed for the initial conditions. In essence we are tracing where stars end up based on their initial actions. In order to make this evident at all times, we subscript actions with a ' $O$ '. Thus our notation for radial, azimuthal and vertical action is $\left(\mathrm{J}_{\mathrm{R}, 0}, \mathrm{~J}_{\phi, 0}, \mathrm{~J}_{\mathrm{z}, 0}\right)$. This does not mean that we are assuming that the actions are conserved during the process of bar and $\mathrm{B} / \mathrm{P}$ bulge formation.

Since, for a finite disc, both the vertical and radial velocity dispersion must decrease radially, both $\mathrm{J}_{\mathrm{z}, 0}$ and $\mathrm{J}_{\mathrm{R}, 0}$ must be correlated with radius, and therefore with $\mathrm{J}_{\phi, 0}$, and possibly with each other. Table 1 lists the Pearson $r$ values of the correlations between all pairs of actions in the various simulations. The largest absolute Pearson $r$ value is 0.42 for the correlation between $\mathrm{J}_{\phi, 0}$ and $\mathrm{J}_{\mathrm{R}, 0}$ in model 5 . The correlations are weakest between $\mathrm{J}_{\mathrm{R}, 0}$ and $\mathrm{J}_{\mathrm{z}, 0}$ in most cases. Frequencies, on the other hand, are much more covariant, with the Pearson $r$ between the radial frequency, $\Omega_{R, 0}$, and the vertical frequency, $\Omega_{z, 0}$, of 0.55 in the fiducial model, and 0.70 in model 5 . For this reason we do not consider the frequencies in any of our analysis.

Part of our analysis consists of splitting the models into quartiles of their action distributions. The ranges of actions in these quartiles for each model are listed in Table 2.

\subsection{Dependence of the bar strength on the actions}

Fig. 4 shows the initial and final density distributions in the lowest and highest quartiles of each action (listed in Table 2). The middle row shows the evolution of the $\mathrm{J}_{\mathrm{R}, 0}$ quartiles. Initially the population with the largest $J_{R, 0}$ is concentrated towards the centre, while those in the lowest 

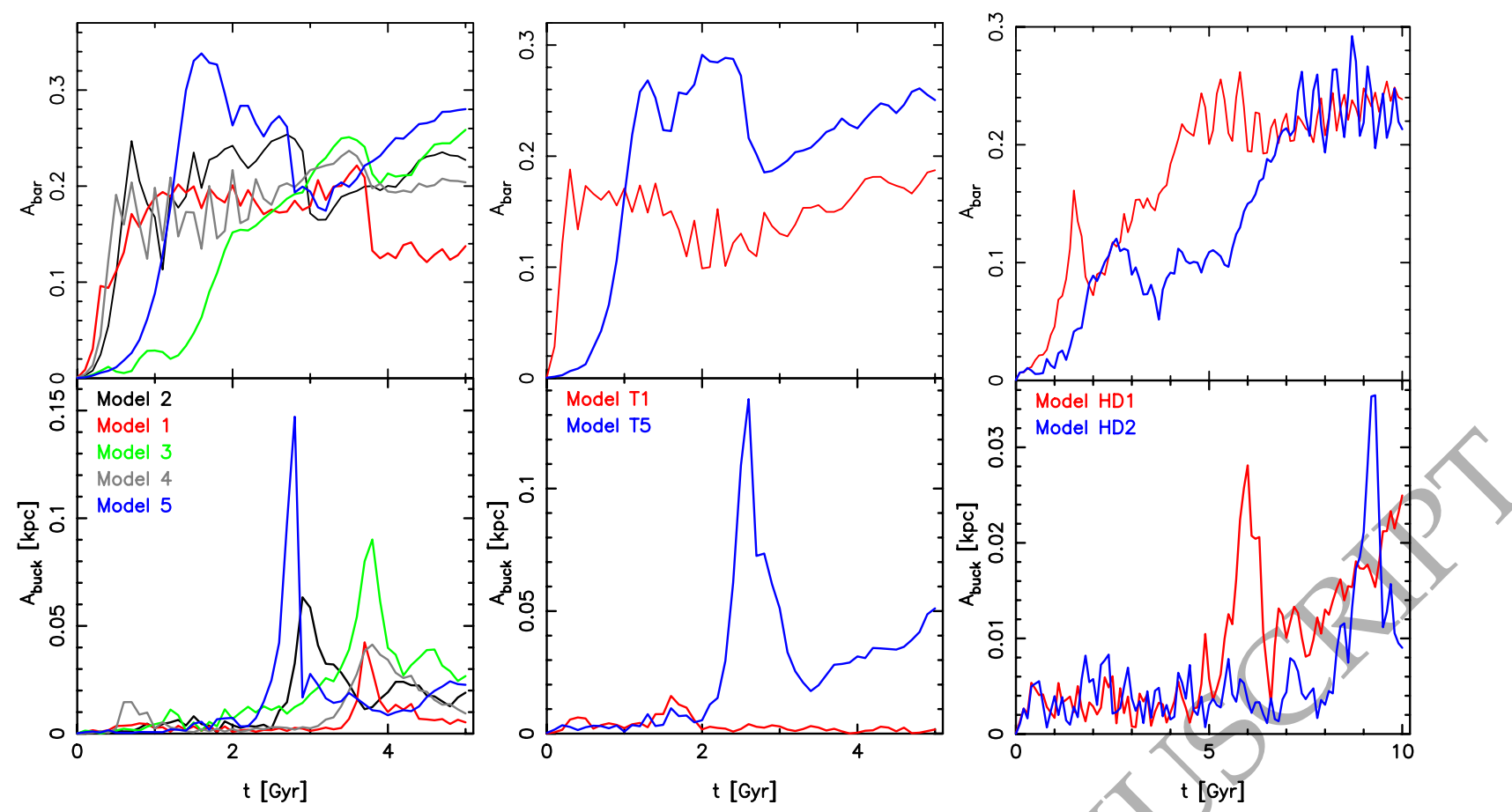

Figure 2. The global bar (top) and buckling (bottom) amplitudes of the simulation suite. Note that both vertical and horizontal axes change between the different panels. All models form a bar and buckle, but there is a wide range of buckling strengths.

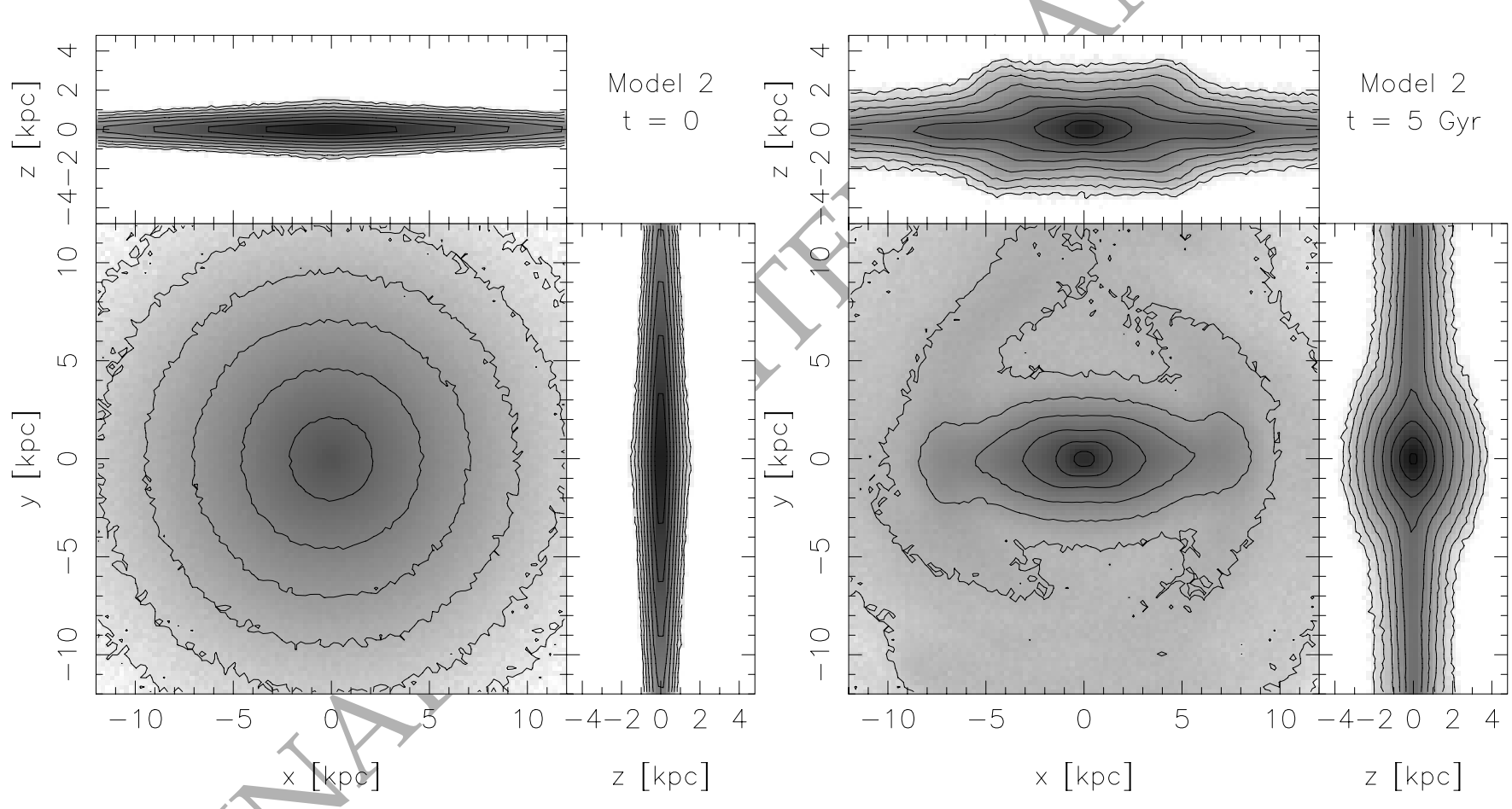

Figure 3. The density distribution of model 2 at $t=0$ (left) and at the end of the simulation, at $t=5$ Gyr, (right) for 3 different projections. The density levels are arbitrary. A prominent bar, with a $\mathrm{B} / \mathrm{P}$-shaped bulge, has formed by $t=5 \mathrm{Gyr}$. The bar has been rotated into the $x$-axis.

$\mathrm{J}_{\mathrm{R}, 0}$ quartile are more radially extended. This continues to be true once the bar forms. At $t=5$ Gyr the bar is visibly stronger in the lowest $\mathrm{J}_{\mathrm{R}, 0}$ quartile. The top row reveals a very similar behaviour for $\mathrm{J}_{\mathrm{z}, 0}$ both before and after the bar forms. Compared with the bar in the $\mathrm{J}_{\mathrm{R}, 0}$ quartiles, the bar is weaker in the lowest $J_{z, 0}$ quartile and stronger at the highest one, i.e. there is a larger range in the bar strength when populations are separated by $\mathrm{J}_{\mathrm{R}, 0}$ than when they are separated by $\mathrm{J}_{z, 0}$. The radial action therefore seems to be a more important factor in determining the bar strength of a 


\begin{tabular}{|c|c|c|c|c|c|}
\hline Model & Action & $\begin{array}{l}1^{s t} \text { quartile } \\
{\left[\mathrm{kpc} \mathrm{km} \mathrm{s}^{-1}\right]}\end{array}$ & $\begin{array}{l}2^{\text {nd }} \text { quartile } \\
{\left[\mathrm{kpc} \mathrm{km} \mathrm{s}^{-1}\right]}\end{array}$ & $\begin{array}{l}3^{r d} \text { quartile } \\
{\left[\mathrm{kpc} \mathrm{km} \mathrm{s}^{-1}\right]}\end{array}$ & $\begin{array}{l}4^{\text {th }} \text { quartile } \\
{\left[\mathrm{kpc} \mathrm{km} \mathrm{s}^{-1}\right]}\end{array}$ \\
\hline 1 & $\mathrm{~J}_{\mathrm{z}, 0}$ & $<1.9$ & $1.9-5.0$ & $5.0-11.5$ & $>11.5$ \\
\hline 2 & $\mathrm{~J}_{\mathrm{z}, 0}$ & $<1.8$ & $1.8-5.0$ & $5.0-11.6$ & $>11.6$ \\
\hline 3 & $\mathrm{~J}_{\mathrm{z}, 0}$ & $<1.8$ & $1.8-4.9$ & $4.9-11.7$ & $>11.7$ \\
\hline 4 & $\mathrm{~J}_{\mathrm{z}, 0}$ & $<0.6$ & $0.6-1.8$ & $1.8-4.2$ & $>4.2$ \\
\hline 5 & $\mathrm{~J}_{\mathrm{z}, 0}$ & $<5.3$ & 5.3-13.9 & $13.9-31.4$ & $>31.4$ \\
\hline $\mathrm{T} 1$ & $\mathrm{~J}_{\mathrm{z}, 0}$ & $<0.7$ & $0.7-2.2$ & $2.2-7.9$ & $>7.9$ \\
\hline T5 & $\mathrm{J}_{\mathrm{z}, 0}$ & $<3.2$ & $3.2-10$ & $10-28$ & $>28$ \\
\hline HD1 & $\mathrm{J}_{\mathrm{z}, 0}$ & $<1.2$ & $1.2-3.3$ & $3.3-7.4$ & $>7.4$ \\
\hline HD2 & $\mathrm{J}_{\mathrm{z}, 0}$ & $<1.2$ & $1.2-3.3$ & $3.3-7.5$ & $>7.5$ \\
\hline 1 & $\mathrm{~J}_{\mathrm{R}, 0}$ & $<4$ & $4-12$ & $12-27$ & $>27$ \\
\hline 2 & $\mathrm{~J}_{\mathrm{R}, 0}$ & $<9$ & $9-27$ & $27-65$ & $>65$ \\
\hline 3 & $\mathrm{~J}_{\mathrm{R}, 0}$ & $<17$ & $17-53$ & $53-130$ & $>130$ \\
\hline 4 & $\mathrm{~J}_{\mathrm{R}, 0}$ & $<9$ & $9-27$ & 27-64 & $>64$ \\
\hline 5 & $\mathrm{~J}_{\mathrm{R}, 0}$ & $<9$ & $9-27$ & $27-67$ & $>67$ \\
\hline $\mathrm{T} 1$ & $\mathrm{~J}_{\mathrm{R}, 0}$ & $<7$ & $7-19$ & $19-44$ & $>44$ \\
\hline T5 & $\mathrm{J}_{\mathrm{R}, 0}$ & $<18$ & $18-48$ & $48-106$ & $>106$ \\
\hline HD1 & $\mathrm{J}_{\mathrm{R}, 0}$ & $<1$ & $1-4$ & 4-9 & $>9$ \\
\hline HD2 & $\mathrm{J}_{\mathrm{R}, 0}$ & $<2$ & $2-7$ & $7-16$ & $>16$ \\
\hline 1 & $\mathrm{~J}_{\phi, 0}$ & $<348$ & $348-822$ & $822-1473$ & $>1473$ \\
\hline 2 & $\mathrm{~J}_{\phi, 0}$ & $<255$ & $255-731$ & $731-1415$ & $>1415$ \\
\hline 3 & $\mathrm{~J}_{\phi, 0}$ & $<163$ & $163-568$ & $568-1315$ & $>1315$ \\
\hline 4 & $\mathrm{~J}_{\phi, 0}$ & $<265$ & $265-736$ & $736-1406$ & $>1406$ \\
\hline 5 & $\mathrm{~J}_{\phi, 0}$ & $<234$ & $234-712$ & $712-1414$ & $>1414$ \\
\hline $\mathrm{T} 1$ & $\mathrm{~J}_{\phi, 0}$ & $<336$ & $336-768$ & $768-1394$ & $>1394$ \\
\hline $\mathrm{T} 5$ & $\mathrm{~J}_{\phi, 0}$ & $<223$ & 223-639 & $639-1282$ & $>1282$ \\
\hline HD1 & $\mathrm{J}_{\phi, 0}$ & $<278$ & $278-626$ & 626-1139 & $>1139$ \\
\hline HD2 & $\mathrm{J}_{\phi, 0}$ & $<258$ & 258-609 & $609-1127$ & $>1127$ \\
\hline
\end{tabular}

Table 2. Action quartiles of the models.

given population than is the vertical action. The third row shows $\mathrm{J}_{\phi, 0}$ which reveals that $\mathrm{J}_{\phi, 0}$ increases with radius, as expected. The lowest $\mathrm{J}_{\phi, 0}$ quartile is almost completely part of the bar at 5 Gyr but is less peanut-shaped viewed edgeon, while less of the highest $\mathrm{J}_{\phi, 0}$ quartile ends up in the bar but is peanut shaped when viewed edge-on.

We define the $m^{\text {th }}$ Fourier amplitude of a population as:

$$
a_{m}(P)=\left|\frac{\sum_{k \in P} m_{k} e^{i m \phi_{k}}}{\sum_{k \in P} m_{k}}\right|,
$$

where particle $k$ is in the population $P$, and $m_{k}$ and $\phi_{k}$ are its mass and azimuthal angle. Fig. 5 shows the dependence of $a_{2}(J)$, the bar strength of action populations, on the actions. In the $\left(\mathrm{J}_{\phi, 0}, \mathrm{~J}_{\mathrm{R}, 0}\right)$ plane a large $a_{2}(J)$ is present at $\log \left(\mathrm{J}_{\phi, 0} /\left[\mathrm{kpckms}^{-1}\right]\right) \simeq 2.8$, beyond which is a spiral, which produces the weak $a_{2}(J)$ in the outer disc, i.e. at large $\mathrm{J}_{\phi, 0}$. In the $\left(\mathrm{J}_{\phi, 0}, \mathrm{~J}_{\mathrm{z}, 0}\right)$ plane $a_{2}(J)$ appears more uniform across $\mathrm{J}_{\mathbf{Z}, 0}$ at fixed $\mathrm{J}_{\phi, 0}$. As a result $a_{2}(J)$ reaches larger values in the $\left(\mathrm{J}_{\phi, 0}, \mathrm{~J}_{\mathrm{R}, 0}\right)$ plane than in the $\left(\mathrm{J}_{\phi, 0}, \mathrm{~J}_{\mathrm{z}, 0}\right)$ plane. In the $\left(\mathrm{J}_{\mathrm{R}, 0}, \mathrm{~J}_{\mathrm{z}, 0}\right)$ plane the bar appears as two branches, one at $\log \left(\mathrm{J}_{\mathrm{z}, 0} /\left[\mathrm{kpc} \mathrm{km} \mathrm{s}^{-1}\right]\right) \sim 1.5$, and the other at $\log \left(\mathrm{J}_{\mathrm{R}, 0} /\left[\mathrm{kpc} \mathrm{km} \mathrm{s}^{-1}\right]\right) \sim 1.7$. The branch at large $J_{\mathrm{z}, 0}$ is comprised of star particles at $R \lesssim 3 \mathrm{kpc}$, while the stars at the large $\mathrm{J}_{\mathrm{R}, 0}$ branch dominate at larger radii.

Fig. 6 shows the radial profiles of the $m=2$ and $m=4$ amplitudes, $a_{2}(R)$ and $a_{4}(R)$, for the different quartiles. The bar is weaker in the highest $\mathrm{J}_{\mathrm{R}, 0}$ quartile. The differences in $a_{2}(R)$ and $a_{4}(R)$ between different $\mathrm{J}_{\mathrm{z}, 0}$ quartiles are less pronounced than in the $\mathrm{J}_{\mathrm{R}, 0}$ quartiles.

\subsection{Mean-action maps}

Fig. 7 shows the distributions of mean actions in the face-on view of the model at $t=0$ and at $t=5 \mathrm{Gyr}$. At $t=0\left\langle\mathrm{~J}_{\phi, 0}\right\rangle$ increases radially outwards, while $\left\langle\mathrm{J}_{z, 0}\right\rangle$ decreases radially. Instead $\left\langle\mathrm{J}_{\mathrm{R}, 0}\right\rangle$ peaks at $R \simeq 3.8 \mathrm{kpc}$. At $t=5 \mathrm{Gyr}\left\langle\mathrm{J}_{\phi, 0}\right\rangle$ is elongated along the major axis of the bar. In contrast, $\left\langle\mathrm{J}_{\mathrm{R}, 0}\right\rangle$ and, to a lesser extent, $\left\langle\mathrm{J}_{\mathrm{z}, 0}\right\rangle$, are elongated along the bar's minor axis at small radii. These orthogonal orientations are due to the fact that stars with small $J_{R, 0}$ or $J_{z, 0}$ are more elongated along the bar than those with large $\mathrm{J}_{\mathrm{R}, 0}$ or $\mathrm{J}_{\mathrm{z}, 0}$ (Fig. 4). The difference in elongations is weaker for $\mathrm{J}_{z, 0}$, and therefore the overall elongation along the $y$-axis is weaker for this action.

Fig. 8 presents maps of the mean actions in the $(x, z)$ plane (i.e. edge-on) at $t=0$ (left panels) and at $t=5 \mathrm{Gyr}$ (i.e. with the bar viewed side-on, middle panels), and in the $(y, z)$ plane at 5 Gyr (i.e. with the bar viewed end-on, right panels). At $t=0\left\langle\mathrm{~J}_{\phi, 0}\right\rangle$ increases in a radial direction, while $\left\langle\mathrm{J}_{\mathrm{z}, 0}\right\rangle$ increases in the vertical direction. The contours of $\left\langle\mathrm{J}_{\mathrm{R}, 0}\right\rangle$ have a more complicated shape, generally increasing in the vertical direction and decreasing in the radial direction. The peak of $\left\langle\mathrm{J}_{\mathrm{R}, 0}\right\rangle$ is at $R \simeq 3.8 \mathrm{kpc}$ off the mid-plane. At the end of the simulation the contours of the $\left\langle\mathrm{J}_{\phi, 0}\right\rangle$ distribution are centred on the origin and are quite vertical. Instead, both $\left\langle\mathrm{J}_{\mathrm{R}, 0}\right\rangle$ and $\left\langle\mathrm{J}_{\mathrm{z}, 0}\right\rangle$ have contours that are pinched 

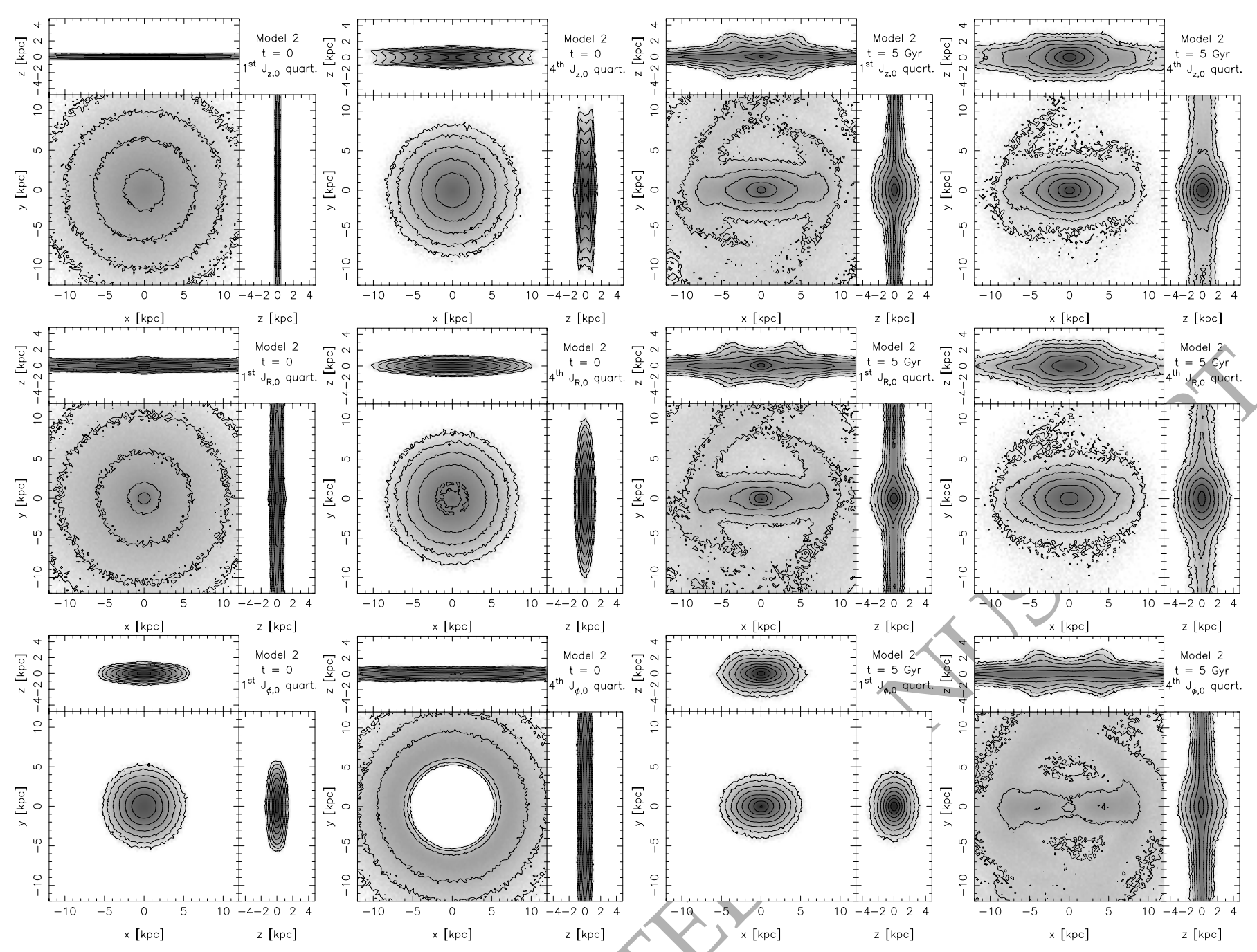

Figure 4. Evolution of the mass distribution in model 2 separated into action quartiles. At top is $\mathrm{J}_{z, 0}$, followed by $\mathrm{J}_{\mathrm{R}, 0}$, while at bottom is $\mathrm{J}_{\phi, 0}$. The first and third columns show the lowest quartile while the second and fourth columns show the highest quartile. The two left columns show $t=0$, while the two right columns show the end of the simulation, at 5 Gyr.

when the bar is viewed side-on. The map of $\left\langle\mathrm{J}_{\mathrm{z}, 0}\right\rangle$ has a peak on the minor axis but with a quite low gradient, and almost vertical contours. The map of $\left\langle\mathrm{J}_{\mathrm{R}, 0}\right\rangle$ also has a peak on the minor axis but the contours are now more peanut-shaped. With the bar seen end-on (right column), the maps of $\left\langle\mathrm{J}_{\phi, 0}\right\rangle$ and $J_{z, 0}$ appear hourglass shaped. The map of $\left\langle J_{R, 0}\right\rangle$ in this projection has a significant vertical gradient and exhibits a "mushroom cap" shape of high $\left\langle\mathrm{J}_{\mathrm{R}, 0}\right\rangle$ at large $|z|$.

\subsection{Vertical profiles in the bulge}

Fig. 8 showed that the formation of the B/P bulge changes the vertical gradients of the actions in the inner galaxy. Fig. 9 explores the evolution of the vertical profiles of the actions in the bulge region, defined as inside $R_{\mathrm{d}}=2.4 \mathrm{kpc}$ (we have confirmed that the trends shown here are similar inside at least $3 R_{\mathrm{d}}$, but this is too large a radius for the $\mathrm{B} / \mathrm{P}$ bulge of some of the other models, such as model 1). The initially vertically declining profile of $\left\langle\mathrm{J}_{\phi, 0}\right\rangle$ is transformed into a rapidly rising profile, which peaks at $|z| \simeq 2 \mathrm{kpc}$, and then declines slowly beyond. The initially monotonically in- creasing $\left\langle\mathrm{J}_{z, 0}\right\rangle$ profile is almost completely flattened by the formation of the $\mathrm{B} / \mathrm{P}$ bulge. Conversely the initially rather flat profile of $\left\langle\mathrm{J}_{\mathrm{R}, 0}\right\rangle$ is transformed by the bar into a monotonically rising profile, indicating that stars with the largest radial motions are the ones that rise to the largest heights.

Fig. 10 shows the vertical density profile for all stars inside $2.4 \mathrm{kpc}$ (black points) and for the same stars separated into low and high action halves. The low and high action profiles are quite parallel, and overlap, for $\mathrm{J}_{\phi, 0}$ and $\mathrm{J}_{z, 0}$. However the vertical profiles of the low and high $\mathrm{J}_{\mathrm{R}, 0}$ diverge, with the density of low $J_{R, 0}$ stars declining more rapidly than that of the high $\mathrm{J}_{\mathrm{R}, 0}$. Above $3 \mathrm{kpc}$ essentially all stars are from the high $\mathrm{J}_{\mathrm{R}, 0}$ half.

\subsection{Bimodal (X-shaped) distributions}

Fig. 11 shows the density distribution of stars at different heights for stars separated into the four quartiles of $\mathrm{J}_{z, 0}$ (left), $\mathrm{J}_{\mathrm{R}, 0}$ (centre) and $\mathrm{J}_{\phi, 0}$ (right) listed in Table 2 . All exhibit bimodal distributions for at least some quartiles. The separations between the peaks at fixed $|z|$ increase with $\mathrm{J}_{\phi, 0}$ 


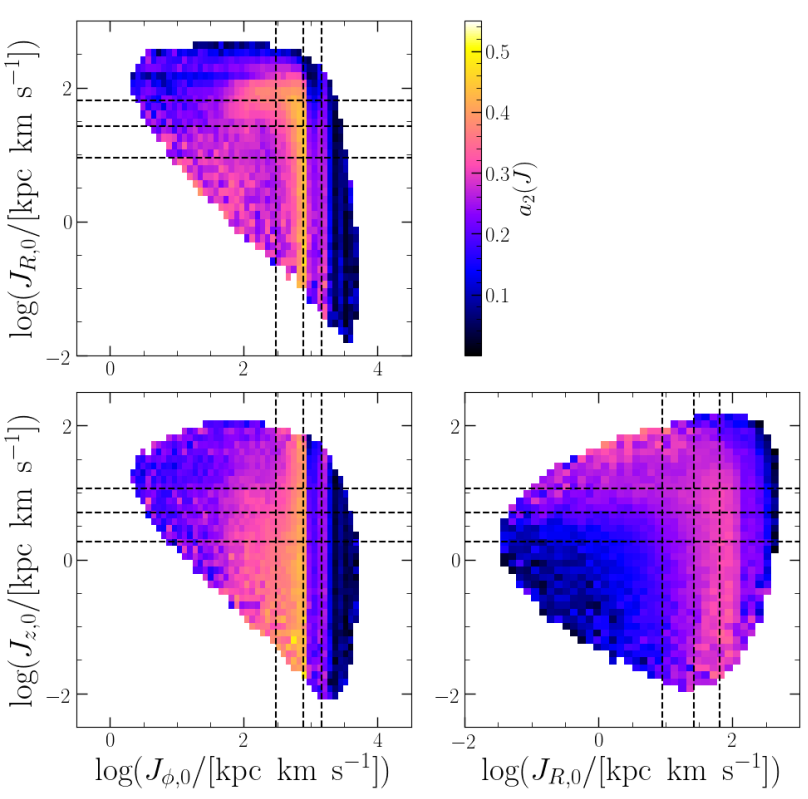

Figure 5. The strength of the $m=2$ Fourier moment, $a_{2}(J)$, in model 2 as a function of the initial actions. Only bins with more than 200 particles are shown. The large values of $a_{2}(J)$ in the region at $\log \left(\mathrm{J}_{\phi, 0} /\left[\mathrm{kpc} \mathrm{km} \mathrm{s}^{-1}\right]\right) \lesssim 2.8$ are produced by the bar, while spirals are responsible for the large values at larger $\mathrm{J}_{\phi, 0}$. The vertical and horizontal dashed black lines separate the different quartiles in the associated actions.

and decrease with increasing $\mathrm{J}_{\mathrm{R}, 0}$ and $\mathrm{J}_{\mathrm{z}, 0}$. Moreover these separations increase with larger $|z|$, i.e. the distributions are $\mathrm{X}$-shaped. The behaviour of the density distributions separated by $J_{R, 0}$ and $J_{z, 0}$ are somewhat similar. The separation appears first in $J_{\phi, 0}$, followed by $J_{z, 0}$, while $J_{R, 0}$ is the last to show the bimodality. Of the three actions, the separation between the peaks is largest in $\mathrm{J}_{\phi, 0}$.

\subsection{Vertical heating and radial cooling}

We define the thickness of stellar populations, $h_{2}$, as the standard deviation of the vertical positions of stars. The left panels of Fig. 12 show the evolution of the thickness in $\left(\mathrm{J}_{\mathrm{R}, 0}, \mathrm{~J}_{\mathrm{z}, 0}\right)$ space. At $t=0$ the thickness is a strong function of $J_{z, 0}$, which merely shows that the actions are calculated properly. The contours of constant $h_{z}$ are not horizontal, as a consequence of the radial dependence of the potential. After the $\mathrm{B} / \mathrm{P}$ bulge forms, at $5 \mathrm{Gyr}$, the thickness increases monotonically with $\mathrm{J}_{\mathrm{R}, 0}$, with the thickest populations those at largest $\mathrm{J}_{\mathrm{R}, 0}$. The thickness increases with $\mathrm{J}_{\mathrm{z}, 0}$ up to a point, but then decreases again, with the peak reached never as high as that in $\mathrm{J}_{\mathrm{R}, 0}$. The map of the change in thickness between the final and initial states, $\Delta h_{z}$, shows that $\Delta h_{z}$ increases most strongly with increasing $\mathrm{J}_{\mathrm{R}, 0}$. Surprisingly vertical heating is negative, i.e. stellar populations become thinner, at the largest $\mathrm{J}_{\mathrm{z}, 0}$. Vertical heating therefore is primarily determined by $\mathrm{J}_{\mathrm{R}, 0}$ and substantially less by $J_{z, 0}$. A comparable figure for the vertical velocity dispersion, $\sigma_{z}$, not shown here, is very similar to the left column of Fig. 12, including having a population with $\Delta \sigma_{z}<0$.

Conversely we expect $\sigma_{R}$ should also decrease in the populations with large $\mathrm{J}_{\mathrm{R}, 0}$. We test this prediction in

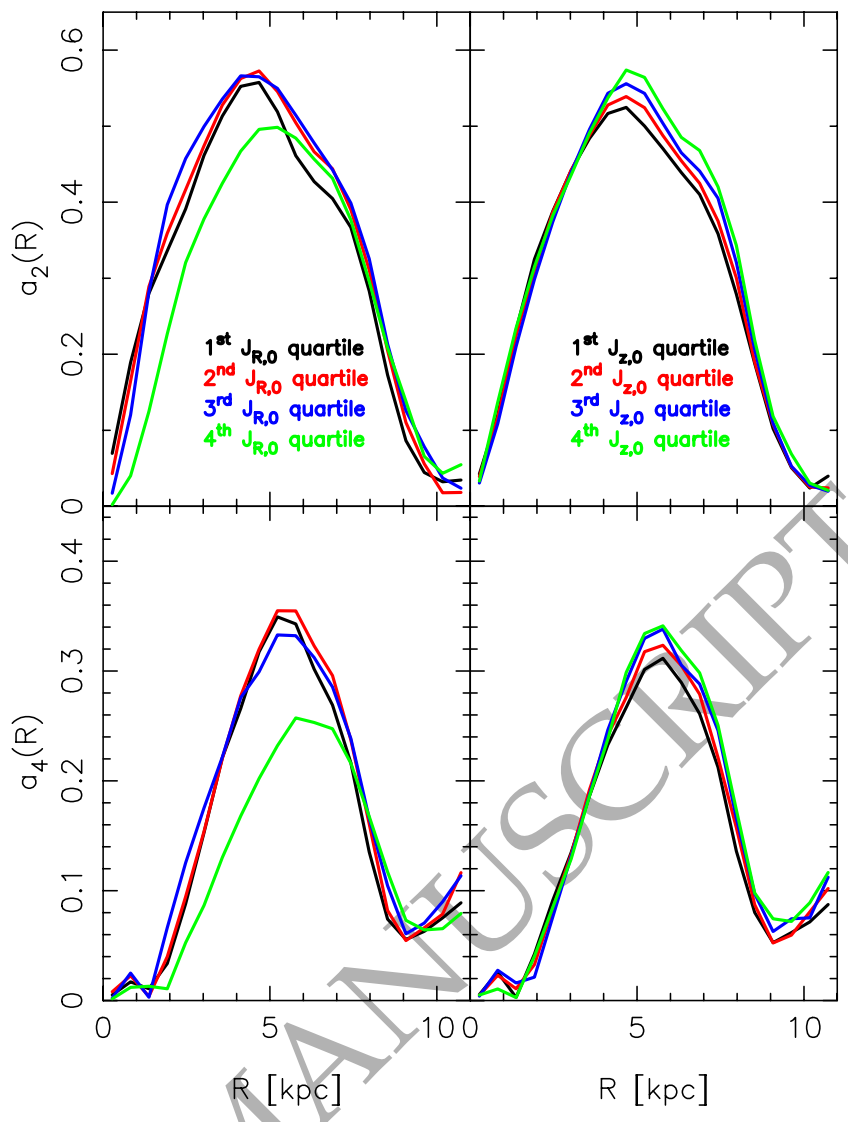

Figure 6. The bar strength as a function of radius for model 2 split into different action quartiles. The top row shows the $m=$ 2 Fourier amplitudes while the bottom row shows the $m=4$ amplitudes. At left the disc is split into $\mathrm{J}_{\mathrm{R}, 0}$ quartiles while at right into $J_{\mathrm{z}, 0}$ quartiles. The bar strength has a larger dynamic range in $J_{R, 0}$ than it does in $J_{z, 0}$.

the right panels of Fig. 12, which show that although most of the system heats radially, all populations at $\log \left(\mathrm{J}_{\mathrm{R}, 0} /\left[\mathrm{kpc} \mathrm{km} \mathrm{s}^{-1}\right]\right) \gtrsim 2.2 \mathrm{cool}$. The contour with $\Delta \sigma_{R}=$ 0 , shown by the dashed grey line, is surprisingly vertical considering that the bar instability increases the radial random motion, driving $\sigma_{R}$ up, and setting up the radial anisotropy which drives the buckling instability (Araki 1985; Merritt \& Sellwood 1994). The fraction of the disc that radially cools is larger than that which cools vertically.

\section{DIFFERENT INITIAL CONDITIONS}

The analysis thus far has considered only the fiducial model 2 . Now we consolidate these results by considering the rest of the simulation suite. The appendices present figures similar to Figs. 3 to 12 for the rest of the models. This section discusses trends that arise from varying the radial random motion, varying the thickness, from thin+thick disc structure and from a high dark matter fraction. Section 4.5 provides a synthesis of the simulation suite. 

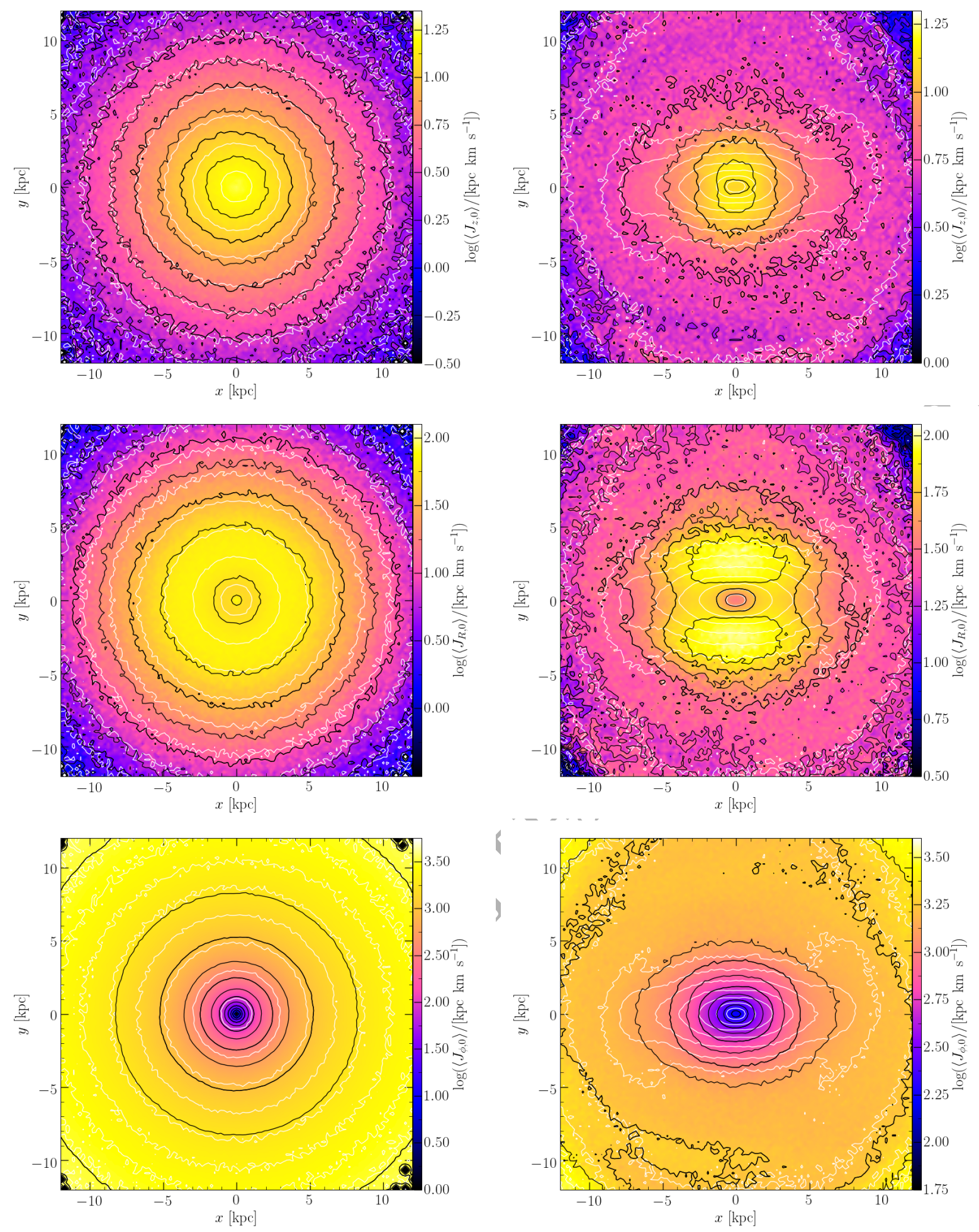

Figure 7. The face-on surface density (white contours) and mean actions (colours and black contours) at $t=0$ (left) and at $t=5 \mathrm{Gyr}$ (right) in model 20

\subsection{Varying the radial velocity dispersion}

Models 1 and 3 are initially radially cooler and hotter, respectively, than the fiducial model 2 . Model 1 has the narrowest range of $\mathrm{J}_{\mathrm{R}, 0}$ of any of the baryon-dominated models (the model has Toomre- $Q \sim 0.8$ over a short radial range).
The bar strength barely varies with $\mathrm{J}_{\mathrm{R}, 0}$ or $\mathrm{J}_{\mathrm{z}, 0}$, and this is the only baryon-dominated model in which the vertical gradient is more prominent in $\mathrm{J}_{\phi, 0}$ than in $\mathrm{J}_{\mathrm{R}, 0}$, reflecting on the quite narrow range of $\mathrm{J}_{\mathrm{R}, 0}$. In the inner $R_{\mathrm{d}}$, the separation of the vertical profiles of the action halves is larger 

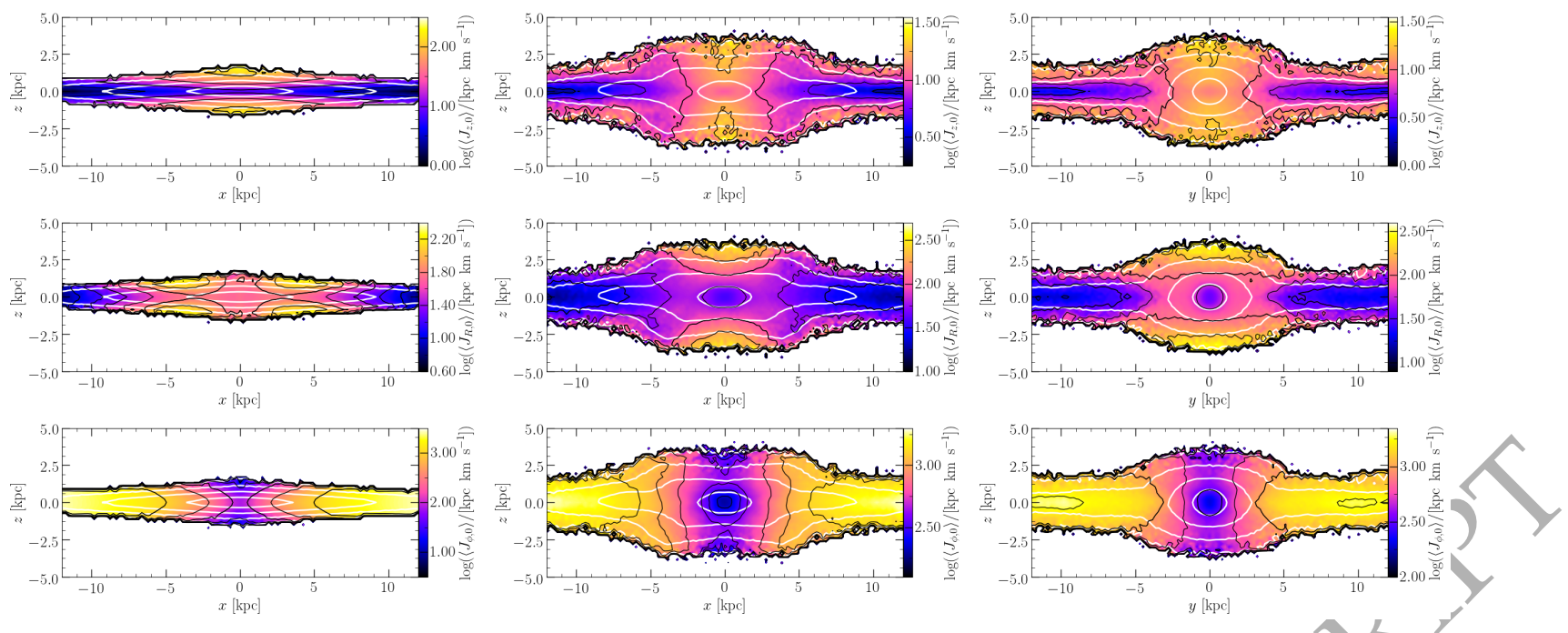

Figure 8. Actions in the $(x, z)$ plane at $t=0$ (left) and at the end of the simulation, at $t=5$ Gyr (middle) and in the (y,z) plane (right) in model 2. From top to bottom we plot $\left\langle\mathrm{J}_{z, 0}\right\rangle,\left\langle\mathrm{J}_{\mathrm{R}, 0}\right\rangle$ and $\left\langle\mathrm{J}_{\phi, 0}\right\rangle$. Particles are selected in the slice $|y|<2 \mathrm{kpc}$ in the two left columns and in the slice $|x|<2 \mathrm{kpc}$ in the right column. Black contours show the actions, while white contours show the density.

in $\mathrm{J}_{\phi, 0}$ than in $\mathrm{J}_{\mathrm{R}, 0}$. In model 3 the bar amplitude depends strongly on $\mathrm{J}_{\mathrm{R}, 0}$ and less so on $\mathrm{J}_{\mathrm{z}, 0}$. In the face-on distributions, the peaks in $\left\langle J_{R, 0}\right\rangle$ on the minor axis of the bar become more prominent as the radial random motion increases. In the side-on view, pinching of $\left\langle\mathrm{J}_{\mathrm{R}, 0}\right\rangle$ is present in all three models. In model 3 the arms of the X-shape become evident at larger height for both $\mathrm{J}_{R, 0}$ and $\mathrm{J}_{z, 0}$. In all three models the separation of peaks in the bimodality is strongest in $\mathrm{J}_{\phi, 0}$, with the highest quartile always being the most widely separated. All three models are thickest, and thicken the most, at the largest $\mathrm{J}_{\mathrm{R}, 0}$, including in model 1 which has the narrowest range of $\mathrm{J}_{\mathrm{R}, 0}$. In contrast populations with the largest $\mathrm{J}_{z, 0}$ always get thinner after the $\mathrm{B} / \mathrm{P}$ bulge develops. In model 3 , the vertical profile of $\left\langle J_{R, 0}\right\rangle$ is still rising at $|z|=5 \mathrm{kpc}$, and the vertical density of the low $\mathrm{J}_{\mathrm{R}, 0}$ half declines rapidly, while that of the high $\mathrm{J}_{\mathrm{R}, 0}$ half dominates (over all action halves) from $|z| \simeq 2 \mathrm{kpc}$.

\subsection{Varying the thickness}

Models 4 and 5 have thinner and thicker initial discs than the fiducial model, respectively. Model 4 thickens considerably, in spite of having one of the mildest bucklings. Its initially low thickness ensures that the strong bar that forms produces a highly anisotropic velocity distribution, leading to a strong vertical heating. Because it starts out so thin, the region of action space where the populations become thinner is reduced compared to the fiducial model, but it is still present. The final vertical profiles of the actions follow the same trend as in the fiducial model. All four $\mathrm{J}_{z, 0}$ quartiles have nearly identical bar amplitude, while a large weakening in the bar strength and peanutiness is evident for the highest $\mathrm{J}_{\mathrm{R}, 0}$ quartile. In the initially thickest disc, in model 5 , the highest $J_{z, 0}$ populations still end up thinner after $B / P$ formation over a more extended region than in the fiducial model. The bar strength and peanutiness is still better separated by $\mathrm{J}_{\mathrm{R}, 0}$, rather than by $\mathrm{J}_{\mathrm{z}, 0}$. The bar amplitudes are similar in all the populations except for the highest $\mathrm{J}_{\mathrm{R}, 0}$ one in model 4 while in model 5 the difference is largely in the radius of the peak amplitude rather than the peak itself. As in models $1-3$, the populations that thicken the most, and end up thickest, are the ones with the largest $\mathrm{J}_{\mathrm{R}, 0}$.

\subsection{Thin+thick disc models}

Model T1 exhibits much the same trends as the fiducial model. The bar is strongest in the lowest quartiles of $\mathrm{J}_{\mathrm{R}, 0}$ and $J_{z, 0}$, with the difference between bar strengths greater when the model is split by $\mathrm{J}_{\mathrm{R}, 0}$ than by $\mathrm{J}_{\mathrm{z}, 0}$. Only a very minor population of model T1 ends up thinner at the end of the simulation. On the other hand a significant population becomes radially cooler.

In model $\mathrm{T} 5$ the population with the largest $\mathrm{J}_{\mathrm{R}, 0}$ has a significantly weaker bar than in any other population. The smaller range of bar strengths in $\mathrm{J}_{\mathrm{z}, 0}$ quartiles compared to $\mathrm{J}_{\mathrm{R}, 0}$ quartiles is particularly clear in this case and the highest $\mathrm{J}_{\mathrm{R}, 0}$ population has $a_{4} \simeq 0$. The vertical gradient in $\left\langle\mathrm{J}_{\mathrm{z}, 0}\right\rangle$ is considerably weakened by bar and $\mathrm{B} / \mathrm{P}$-bulge formation whilst, once more, an enhanced gradient in $\left\langle\mathrm{J}_{\mathrm{R}, 0}\right\rangle$ develops. Fig. 13 shows the final vertical density profile of model T5 in the inner disc, as well as the same stars separated into halves of $\mathrm{J}_{\mathrm{R}, 0}$, and into those from the thin and thick discs. At large heights, stars are more likely to have originated from the higher $J_{R, 0}$ population than they are from the thick disc. Even when there is a thin and a thick disc dichotomy to start with, the population that ends up thickest, and thickens the most, is still the one with the largest $J_{R, 0}$. As in the fiducial model, a significant population cools vertically, while a different, more sizeable, population cools radially.

\subsection{Dark-matter dominated systems}

The properties of model HD1 are similar to those of model 1. What these two models have in common is a quite narrow range of $\mathrm{J}_{\mathrm{R}, 0}$ (see Table 2 ). As in model 1 , the vertical profiles of the action halves in the inner galaxy are more separated in $\mathrm{J}_{\phi, 0}$ than in $\mathrm{J}_{\mathrm{R}, 0}$. In addition, while the formation of 

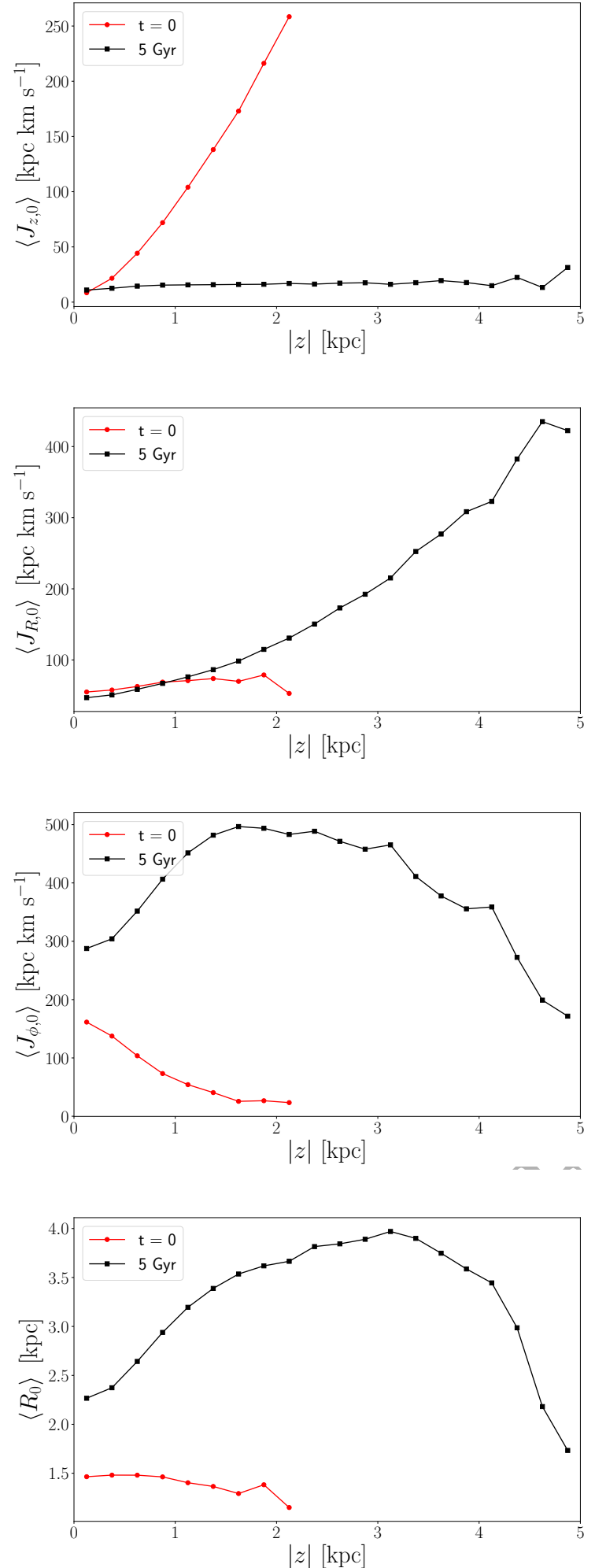

Figure 9. The change in the vertical profiles of $\left\langle\mathrm{J}_{\mathrm{z}, 0}\right\rangle$ (top row), $\left\langle\mathrm{J}_{\mathrm{R}, 0}\right\rangle$ (second row), $\left\langle\mathrm{J}_{\phi, 0}\right\rangle$ (third row), and $\left\langle R_{0}\right\rangle$ (fourth row) in model 2. The initial conditions are shown as (red) filled circles while the final distribution is shown as (black) filled squares. All quantities have been computed within the inner $R_{\mathrm{d}}=2.4 \mathrm{kpc}$.

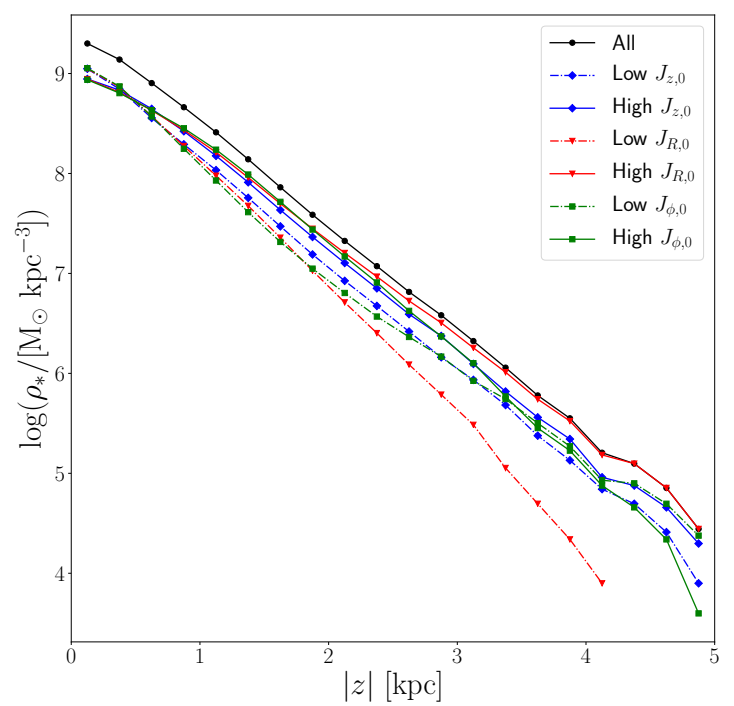

Figure 10. The central vertical density profile of stars in model 2 at $t=5 \mathrm{Gyr}$ split into two equal samples of low and high actions, as indicated. The full sample of stars is shown by the black line. The profiles have been computed within the inner $R_{\mathrm{d}}=2.4 \mathrm{kpc}$.

the $\mathrm{B} / \mathrm{P}$ bulge retains a vertical gradient in $\mathrm{J}_{\mathrm{R}, 0}$, the resulting profile is shallower than at the start. Another similarity between the two models is that the X-shape has comparable strength in all the action quartiles at large heights from the mid-plane. Additionally the bar amplitude is essentially identical in all $\mathrm{J}_{\mathrm{R}, 0}$ and $\mathrm{J}_{\mathrm{z}, 0}$ quartiles. The two peaks are clearly separated, regardless of which action is considered. While a population that cools vertically is readily apparent, unlike model 1 only a very insignificant population cools radially. Model HD2 has a minimum Toomre- $Q \sim 1.2$, but it still has a narrower range of $\mathrm{J}_{\mathrm{R}, 0}$ than does model 1 . While in most respects it behaves like model 2 , the different quartiles have very similar bar amplitudes, while the $\mathrm{X}$-shape is very similar across all the quartiles. Unlike model HD1, in model HD2 a steeper vertical gradient in $\mathrm{J}_{\mathrm{R}, 0}$ develops.

\subsection{Synthesis of the simulation suite}

Overall the simulations exhibit a number of important commonalities. The dominant role of the radial action in predicting the final distributions of stars in the $\mathrm{B} / \mathrm{P}$ bulge is attested by the monotonically increasing vertical heating with increasing $\mathrm{J}_{\mathrm{R}, 0}$, the monotonic vertical profile of $\left\langle\mathrm{J}_{\mathrm{R}, 0}\right\rangle$ within the $\mathrm{B} / \mathrm{P}$ bulge, and the larger separation in bar strengths when stellar populations are separated by $\mathrm{J}_{\mathrm{R}, 0}$. All the models end up thickest in the populations with the largest $\mathrm{J}_{\mathrm{R}, 0}$. In all models a bimodal distribution, i.e. an $\mathrm{X}$ shape, is present at some distance away from the mid-plane; the separation is largest at the highest $\mathrm{J}_{\phi, 0}$ and in the lowest $J_{R, 0}$ and $J_{z, 0}$. The vertical density profiles of the stars in the $J_{\phi, 0}$ and $J_{z, 0}$ halves are quite parallel, but diverge in the $\mathrm{J}_{\mathrm{R}, 0}$ halves. The only models that diverge significantly from these trends are the ones with a narrow range of $J_{R, 0}$, which happens when the initial disc dips below $Q=1$. 


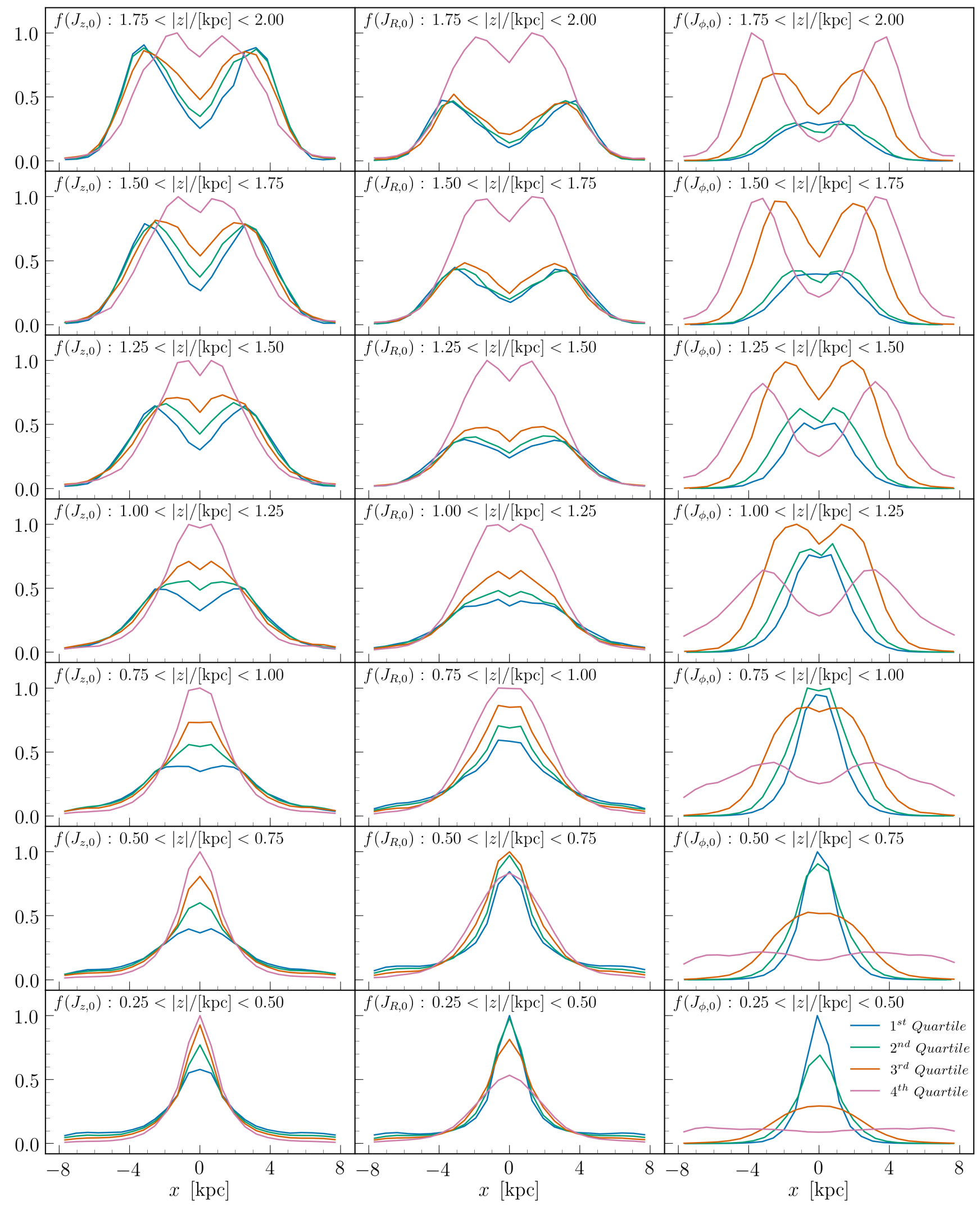

Figure 11. Density profiles along the $x$-axis (along which the bar is aligned) in model 2 at the end of the simulation, $t=5$ Gyr, for different heights above the mid-plane. At each height, the profiles are split into quartiles of the initial actions, as indicated in the top row, with the $1^{\text {st }}$ quartile having the lowest values. Only particles at $|y|<2 \mathrm{kpc}$ are included. From left to right are shown the vertical, radial and azimuthal actions. 

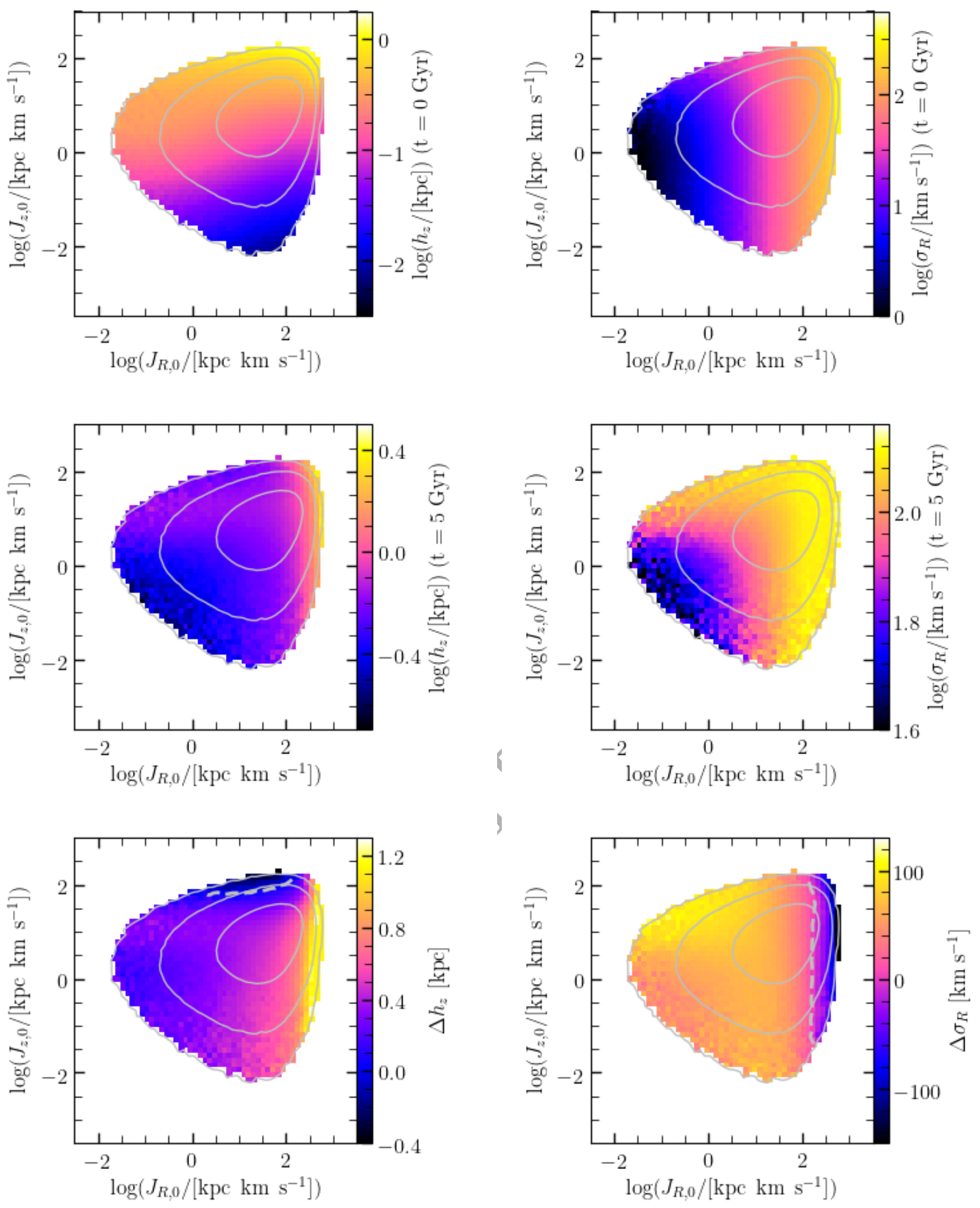

Figure 12. Left: Evolution of $h_{z}$, the thickness of stellar populations, in the $\left(\mathrm{J}_{\mathrm{R}, 0}, \mathrm{~J}_{\mathrm{z}, 0}\right)$ plane of model 2. Right: Evolution of $\sigma_{R}$, the radial velocity dispersion of stellar populations, in the $\left(\mathrm{J}_{\mathrm{R}, 0}, \mathrm{~J}_{\mathrm{z}, 0}\right)$ plane of model 2 . The top row shows $t=0$ and the middle row shows the end of the simulation, $t=5 \mathrm{Gyr}$, both on a log scale. The bottom row shows the difference, now on a linear scale. At large $\mathrm{J}_{z, 0}$ $\Delta h_{z}<0$ i.e. the stellar populations become thinner after the $\mathrm{B} / \mathrm{P}$ bulge forms (bottom left). Similarly, at large $\mathrm{J}_{\mathrm{R}, 0}, \Delta \sigma_{R}<0$, i.e. the stellar populations become radially cooler (bottom right). The dashed grey contours indicate $\Delta h_{z}=0$ (bottom left) and $\Delta \sigma_{R}=0$ (bottom right). Solid contours indicate the density of stars and are spaced by factors of 10, with the peak set at 10,000 particles. 


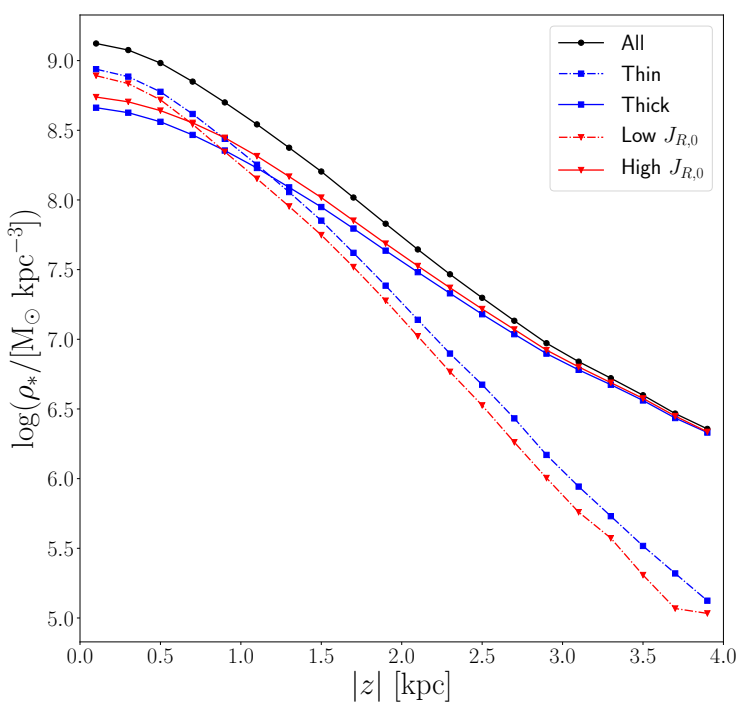

Figure 13. The vertical density profile of stars within $R_{\mathrm{d}}=$ $2.4 \mathrm{kpc}$ at $t=5 \mathrm{Gyr}$ in model T5. The full profile (black) is split into thin and thick discs (blue) and into low and high halves of $\mathrm{J}_{\mathrm{R}, 0}$ (red).

\section{STELLAR POPULATION MODELLING}

The chemistry of bulges provides an important constraint on their formation. There are several observational trends that models of $\mathrm{B} / \mathrm{P}$ bulges need to satisfy. Firstly, in the MW, the density bimodality, i.e. the $\mathrm{X}$-shape, is observed to be pronounced in metal-rich stars and weak or absent in the metal-poor ones (Ness et al. 2012; Uttenthaler et al. 2012; Rojas-Arriagada et al. 2014). Secondly, but related to this, self-consistent chemo-hydrodynamical simulations (Debattista et al. 2017, 2019; Athanassoula et al. 2017) have predicted more pinched metallicity distributions than the density itself when viewed edge-on; this distribution has been confirmed in real galaxies (Gonzalez et al. 2017). This trend is the equivalent of the metallicity dependence of the $\mathrm{X}$ shape, generalised to external galaxies. Lastly, along the MW's minor (vertical) axis, a metallicity gradient is observed (Gonzalez et al. 2011, 2013; Ness et al. 2013; Zoccali et al. 2017).

In order to explore the relation between the action trends found above and the metallicity trends, we now develop a method for assigning metallicity to star particles based on their actions. We employ a self-consistent star-forming chemo-hydrodynamical simulation to determine how the metallicity depends on the actions in an unbarred galaxy. We then use actions to map metallicity from the star-forming simulation to the star particles in the fiducial, pure $N$-body, model. Since the metallicity of stars in real galaxies depends, if anything, on the actions before the bar forms, our emphasis here on the initial actions is wellmatched to the problem at hand. First, however, we consider the limitations of modelling the metallicity based on only single actions to motivate full three-action modelling.

\subsection{Metallicities based on single actions}

\subsubsection{Metallicity based on $\mathrm{J}_{\phi, 0}$ or initial radius}

Martinez-Valpuesta \& Gerhard (2013) investigated the origin of the MW's vertical metallicity gradient in pure $N$-body simulations by assuming that the initial axisymmetric disc had a steep radial metallicity gradient. They showed that in this case, a metallicity gradient similar to the one observed arises. Consistent with this result, the vertical profile of the mean initial radius, $\left\langle R_{0}\right\rangle$, shown in the bottom panel of Fig. 9 , has a shallow declining profile at $t=0$, which is transformed into a rising profile, with a decreasing gradient. The same happens in the full simulation suite but in many cases the profile of $\left\langle R_{0}\right\rangle$ reaches a peak and then declines again. These profiles are very similar to the profiles of $J_{\phi, 0}$, and assigning metallicity by radius can be considered comparable to assignment by the angular momentum.

Assuming metallicity that declines with increasing $\mathrm{J}_{\phi, 0}$ results in an X-shape that is stronger in metal-poor stars than in metal-rich ones. Liu et al. (in progress), reach a similar conclusion. This can be seen in Fig. 11, which shows that it is the populations with largest $J_{\phi, 0}$ which are most widely separated, which is opposite to the trend observed in the MW. Assigning metallicity by $\mathrm{J}_{\phi, 0}$, or nearly equivalently, by radius, therefore produces the wrong $\mathrm{X}$-shape trends compared to those observed in the MW.

\subsubsection{Metallicity based on $\mathrm{J}_{\mathrm{z}, 0}$ or initial height}

Assigning metallicity by $\mathrm{J}_{z, 0}$ is similar to assuming an initial vertical metallicity gradient (although $J_{z, 0}$ is a better measure of a population's thickness than the instantaneous height). Assuming metallicity depends on $J_{z, 0}$ produces an X-shape which is better traced by metal-rich stars, and metallicity distributions that are pinched. For the entire simulation suite we observe that the initial vertical gradient in $J_{z, 0}$ is substantially flattened by the time the $B / P$ bulge forms. Any vertical metallicity gradients are therefore substantially weakened and unlikely to be preserved. An observational test of metallicity depending primarily on $J_{z, 0}$ is that unbarred galaxies should have steeper vertical gradients than barred ones. Since this appears not to be the case (Molaeinezhad et al. 2017), we conclude that $\mathrm{J}_{\mathrm{z}, 0}$ (or height) is not a reliable way of assigning metallicities to particles.

\subsubsection{Metallicity based on $\mathrm{J}_{\mathrm{R}, 0}$ or age}

$\mathrm{J}_{\mathrm{R}, 0}$ measures the radial random motion; stars are generally born on nearly circular orbits and slowly heat radially. Therefore $\mathrm{J}_{\mathrm{R}, 0}$ might be used as a proxy for age, particularly for thin discs. Assuming older stars are more metalpoor would allow metallicity to be assigned based on $\mathrm{J}_{\mathrm{R}, 0}$. This produces most of the trends observed in $\mathrm{B} / \mathrm{P}$ bulges, including a stronger $\mathrm{X}$-shape in metal-rich stars, a vertical metallicity gradient, which can be stronger than in unbarred galaxies, and a pinched metallicity distribution when observed edge-on. The vertical metallicity gradient arises naturally via the monotonically rising profile of $\mathrm{J}_{\mathrm{R}, 0}$ produced by the buckling. Molaeinezhad et al. (2017) find some evidence for a steeper metallicity gradient in barred galaxies than in unbarred galaxies. 

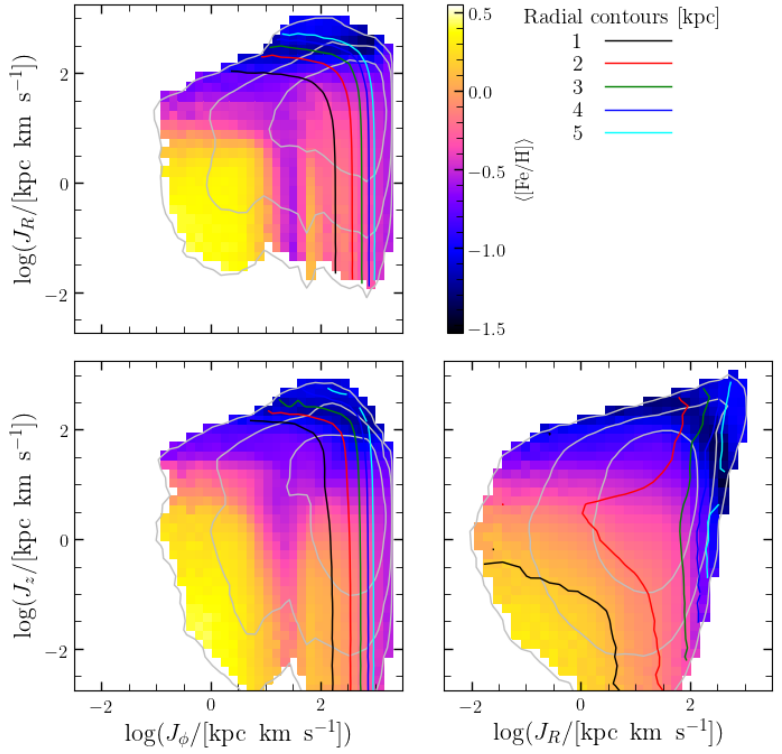

Figure 14. The mean metallicity of the star-forming simulation at 3 Gyr in action space. Coloured contours show the mean radii of particles, as indicated. Solid grey contours indicate the density of stars and are spaced by factors of 10, with the peak set at 10,000 particles. Only bins with more than 100 particles are shown.

The principal drawback of using $J_{R, 0}$ for metallicity modelling is that a single value of $\mathrm{J}_{\mathrm{R}, 0}$ corresponds to a wide range of $\mathrm{J}_{\phi, 0}$, with often very different bar strengths (see Fig. 5). Moreover, in our simulation suite, $\mathrm{J}_{\mathrm{R}, 0}$ does not peak at the centre of the galaxy at $t=0$ (see Fig. 7), which would require that the metallicity peaks away from the centre. Furthermore, the peak of $\mathrm{J}_{\mathrm{R}, 0}$ is generally off the mid-plane (see Fig. 8). Therefore a metallicity based only on $\mathrm{J}_{\mathrm{R}, 0}$ is unlikely to be realistic.

\subsubsection{Metallicity based on radial and vertical position}

Bekki \& Tsujimoto (2011) used the radial and vertical position of a star particle in the initial axisymmetry system to assign metallicity. Using the prescription of Bekki \& Tsujimoto (2011), we find that the X-shape has comparable strength in each metallicity population, contrary to what is observed in the MW. Fundamentally the problem is that all star particles at a given position are treated the same when they should have different ages, and therefore different $\mathrm{J}_{\mathrm{R}, 0}$ and different metallicities.

We therefore conclude that assigning metallicities based on actions requires all three actions. Next we develop a method for such modelling.

\subsection{Three-action metallicity modelling}

\subsubsection{A star-forming donor}

The star-forming model we use to connect actions and metallicities is a generic example of the many MW-mass models we have constructed and presented in the past (e.g. Roškar et al. 2008; Loebman et al. 2011, 2016) but with the $5 \times$ higher resolution used in Portaluri et al. (2017). It evolves self-consistently, with all stars forming out of gas cooling off a hot corona. We use a slightly higher supernova feedback efficiency than in those earlier works, at $4 \times 10^{50}$ erg per supernova. All other parameters are identical to the earlier works and will not be described here, but we anticipate describing the evolution of this simulation in detail elsewhere.

We continue to compute actions using AGAMA (Vasiliev 2019), assuming the model is axisymmetric and using the Stäckel fudge approximation. While the model lacks a bar, it has prominent spirals; as a result, the measured actions are disturbed by the spiral structure. We compare with the actions 100 Myr later, during which time the stars drift with respect to the spirals, and quantify the changes via $d(\mathrm{~J}) \hat{=}$ $F W H M(\Delta \mathrm{J}) /\langle\mathrm{J}\rangle$, where $F W H M(\Delta \mathrm{J})$ is the full width at half-maximum of the change in a particular action. We find changes of $d\left(\mathrm{~J}_{\mathrm{R}}\right)=0.262, d\left(\mathrm{~J}_{\phi}\right)=0.048$ and $d\left(\mathrm{~J}_{\mathrm{z}}\right)=0.013$. We do not attempt to account for these variations in our action-based metallicity mapping.

We use this simulation strictly to query the chemistry as a function of the actions. We consider the model at $t=$ $3 \mathrm{Gyr}$, on the premise that this is a plausible time for when the bar of the MW formed. Fig. 14 shows the density and mean metallicity of the star-forming model in the action space at this time. The density distribution in the $\left(\mathrm{J}_{\mathrm{R}}, \mathrm{J}_{\mathrm{z}}\right)$ plane is qualitatively similar to that in the $\left(\mathrm{J}_{\mathrm{R}, 0}, \mathrm{~J}_{\mathrm{z}, 0}\right)$ plane of the fiducial model (Fig. 12). We refer to this model from here on as the "donor" model.

\subsubsection{Metallicity assignment from the actions}

We now demonstrate assigning metallicities to star particles in the fiducial $N$-body model 2 (hereafter "the target") using the metallicities of star particles in the the donor. Because the density distribution, and the actions, in these two models are quite different, we cannot assign metallicities by directly matching the actions. We start by cutting the donor to only those particles within the volume $|x|,|y| \leqslant 12 \mathrm{kpc}$ and $|z| \leqslant 6 \mathrm{kpc}$. We then construct the cumulative distribution functions, $F_{d, \phi}, F_{d, R}$ and $F_{d, z}$, for $\log \left(\mathrm{J}_{\mathrm{R}}\right), \log \left(\mathrm{J}_{\mathrm{z}}\right)$ and $\log \left(\left|J_{\phi}\right|\right)$. (Here the subscript 'd' refers to the donor model.) After trimming their lower and upper $1 \%$, we compute the average and standard deviation of the metallicity in bins in the $F_{d, \phi} \otimes F_{d, R} \otimes F_{d, z}$ space of the donor. We then use the actions of stars in the target to construct its cumulative distribution functions of the actions $F_{t, \phi}, F_{t, R}$ and $F_{t, z}$ (where now the subscript ' $t$ ' refers to the target model and we again use log of the actions). We then assign a metallicity to star particles in the target by matching the cumulative distribution functions in the donor and the target, $\mathbf{F}_{\mathbf{t}}=\mathbf{F}_{\mathbf{d}}$, by drawing a value of $[\mathrm{Fe} / \mathrm{H}]$ from the Gaussian describing that action bin.

The resulting metallicity maps for the target (fiducial) model at $t=5$ Gyr are shown in Fig. 15. Both edgeon views bear strong resemblance to the metallicity maps of the barred star-forming simulation of Debattista et al. (2017) (their Figure 26). The metallicity distribution is more pinched than the density in the side-on view. Along the vertical axis the metallicity has a declining profile. In order to compare the metallicity gradient with the MW's, the model 

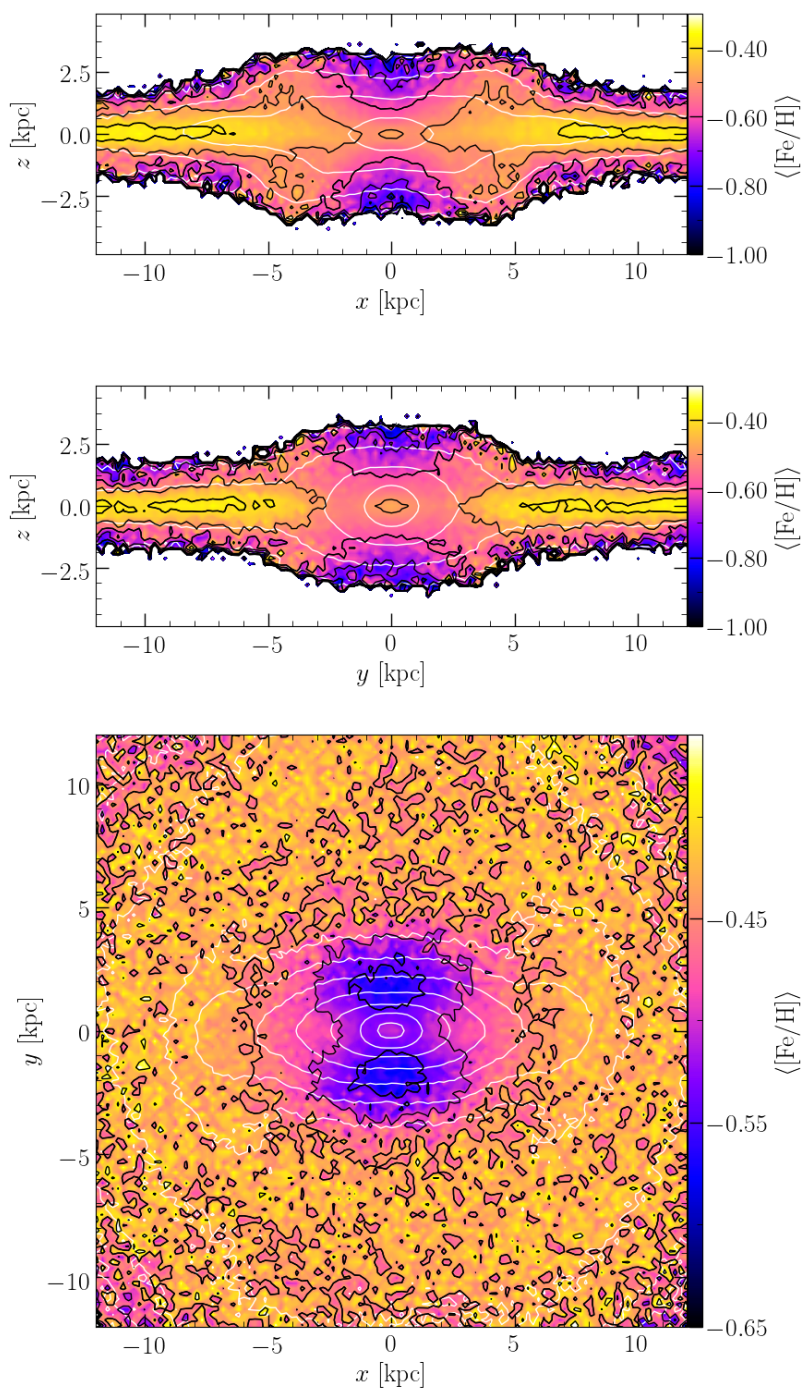

Figure 15. Maps of the mean metallicity of target model 2 at $t=5$ Gyr based on metals mapped using the three actions as described in Section 5.2.2. Top: Edge-on view with the bar seen side-on. Middle: Edge-on view with the bar seen end-on. Bottom: Face-on view. Black contours show $\langle[\mathrm{Fe} / \mathrm{H}]\rangle$ while white contours show the density.

needs to be rescaled because its bar is much longer ${ }^{1}$. Assuming the MW has a bar of $5 \mathrm{kpc}$ (Wegg et al. 2015), we obtain a metallicity gradient of $-0.12 \mathrm{dex} / \mathrm{kpc}$, about half the gradient measured by Gonzalez et al. (2013). Considering that this model is very different from the MW, this is a quite promising result (compared, for instance, with the much weaker vertical gradients in the pure disc simulation of Bekki \& Tsujimoto 2011) that implies that the actions can be successfully used to assign metallicities to particles of $\mathrm{B} / \mathrm{P}$ bulges in $\mathrm{N}$-body simulations.

The metallicity in the face-on view has hollows on the

1 We remind the reader that $N$-body simulations can always be rescaled this way. If length is rescaled as $L^{\prime}=\alpha L$, then the time needs to be rescaled as $T^{\prime}=\alpha^{3 / 2} T$. In our case, with $\alpha=5 / 9$, time is rescaled by a factor of 0.4 . minor axis of the bar, which are not observed in real galaxies. In real galaxies these regions are often found to have "star formation deserts" (SFDs) (James \& Percival 2016, 2018) with ages older than the average. Stellar populations in the SFDs are slowly refilled by stars trapped by the bar at a later stage (Donohoe-Keyes et al. 2019). Since our $N$-body model lacks significant late time bar growth, the presence of these hollows in our model is perhaps not surprising. For our fiducial model, the $[\mathrm{Fe} / \mathrm{H}]$ hollow is only $\sim 0.1$ dex deep, and may be relatively difficult to detect.

\section{DISCUSSION}

Our primary goals in this paper have been to map the initial actions of an axisymmetric disc into the properties of the $\mathrm{B} / \mathrm{P}$ bulge that forms via the bar, and to develop a simple action-based method to assign metallicity to disc particles in order to compare with metallicity trends observed in $\mathrm{B} / \mathrm{P}$ bulges in general, and the MW in particular. We have demonstrated a successful metallicity mapping technique. The study of the role of actions in the development of $\mathrm{B} / \mathrm{P}$ bulges leads to several insights.

\subsection{A statistical mechanical view}

The simplest result to acknowledge is just how reliable the initial actions are at predicting the final distributions of populations in the $\mathrm{B} / \mathrm{P}$ bulge. The bar and buckling instabilities are violent processes (at least in these simulations), during which angular momentum is redistributed, strong spirals propagate, and the bar first grows exponentially in strength, then weakens abruptly. During this time resonances must be sweeping the phase space as the bar assembles and then weakens. Orbital analyses of bars formed in self-consistently evolving simulations typically find resonances well populated (Valluri et al. 2016; Abbott et al. 2017). In most of the simulations presented here the disc potential dominates over the dark halo and the gravitational perturbations must therefore be large. Yet in spite of all this roiling turmoil the structure that emerges in the $\mathrm{B} / \mathrm{P}$ is predetermined by the initial actions. Since energy is being transformed between in-plane and vertical motions, the actions cannot be conserved. The clear dependence on the initial conditions indicates that the dynamical instabilities are not violent enough for complete mixing to occur. Testing this is beyond our scope here, but is worth investigating.

And yet we should not permit ourselves to uncritically accept that the bar+buckling instabilities generally occur in nature with the full vigour of our pure $N$-body simulations. It seems implausible that galaxies often find themselves in a state so unstable that a substantial reorganisation of their states begins immediately, and with vigour. Indeed simulations that include gas find bars forming more slowly and buckling much more gently (Berentzen et al. 1998; Debattista et al. 2017), even in a cosmological context (Debattista et al. 2019). The consequent energy redistribution that must occur is probably milder.

Nonetheless, nature presents examples of buckling bars (Erwin \& Debattista 2016; Li et al. 2017), including in the presence of gas. Whether these are events provoked by external perturbations, or driven by purely internal secular 
evolution is as yet unclear. However a simple model of bar and $\mathrm{B} / \mathrm{P}$-bulge formation that assumes rapid violent buckling is able to reproduce the observed fraction of $\mathrm{B} / \mathrm{P}$ bulges and buckling bars (the known number of which is still less than 10) amidst barred galaxies (Erwin \& Debattista 2016). Complicating this simple picture is the observation that the fraction of barred galaxies that host $\mathrm{B} / \mathrm{P}$ bulges rises rapidly at $\log \left(M_{*} / \mathrm{M}_{\odot}\right) \gtrsim 10.4$, with no evidence that the (present day) gas fraction matters (Erwin \& Debattista 2017). Even more puzzling, this transitional mass appears unchanged since redshift $z \simeq 1$ (Kruk et al. 2019). Clearly there is still much to learn about $\mathrm{B} / \mathrm{P}$ bulge formation and more detailed study of stellar populations in the MW, and in external galaxies, may provide clues to a better understanding.

\subsection{Kinematic fractionation}

Building on the work of Merritt \& Sellwood (1994), Debattista et al. (2017) argued that the vertical evolution of B/P bulges is driven by the ability of stars to respond in phase with the vertical forcing from a bending bar (through the buckling instability). Stars support a vertical perturbation of $m=2$ form so long as they satisfy the condition:

$$
\Omega_{z}>2\left(\Omega_{\phi}-\Omega_{p}\right),
$$

where $\Omega_{z}$ and $\Omega_{\phi}$ are the vertical and rotational frequencies of the star, respectively, and $\Omega_{p}$ is the pattern speed of the bar. Debattista et al. (2017) demonstrated that $\Omega_{z}$ declines rapidly with the amplitude of the vertical excursion, $z_{\max }$, whereas $\Omega_{\phi}$ does not. The separation of stellar populations by the bar occurs because the larger asymmetric drift (lower $\Omega_{\phi}$ ) of stars with larger radial random motions allows them to remain in tune with the vertical forcing by the bar to greater heights. This allows the star to gain vertical energy from the bending wave until eventually it is deposited at a $z_{\max }$ where the condition of Eqn. 8 is no longer satisfied. This $z_{\max }$ is larger for stars with larger radial action because of their lower $\Omega_{\phi}$. For this reason, they proposed that the radial random motion of stars determined the vertical height stars can reach during buckling, with radially hotter stars reaching larger heights.

Recently, Di Matteo et al. (2019) argued that the vertical dispersion before the bar forms is just as important as the radial dispersion. We have shown here that the vertical thickening of populations is a strongly monotonic function of $\mathrm{J}_{\mathrm{R}, 0}$, but depends less strongly on $\mathrm{J}_{\mathrm{z}, 0}$. Indeed we have seen that the populations with the largest $\mathrm{J}_{z, 0}$ generally become thinner after the formation of a $\mathrm{B} / \mathrm{P}$ bulge, and this population can be significant when the initial disc is thick. A monotonically increasing vertical profile of $\left\langle\mathrm{J}_{\mathrm{R}, 0}\right\rangle$ develops in most modéls as a result of $\mathrm{B} / \mathrm{P}$ formation, while the vertical gradient in $\left\langle J_{z, 0}\right\rangle$ is largely erased. Both these facts argue that the radial motions are much more important for determining the final vertical thickening of a population, as proposed by Debattista et al. (2017), and at odds with the suggestion of Di Matteo et al. (2019). Nevertheless, a thick, vertically hot, disc is also likely to be radially hot; as such the combination of a thin and a thick disc is still likely to retain some of the vertical gradient inherent in the different metallicities of the two discs once a bar forms. The physical mechanism driving the final metallicity gradient remains kinematic fractionation acting on stellar populations with different radial actions, not on the vertical actions.

\subsection{Insights from bulge chemistry}

The vertical metallicity gradient (Zoccali et al. 2008; Gonzalez et al. 2011; Johnson et al. 2011, 2013) and the metallicity dependence of the X-shape (Ness et al. 2012; Uttenthaler et al. 2012; Rojas-Arriagada et al. 2014) constrain the formation of the MW's bulge. Bekki \& Tsujimoto (2011) argued that, in single disc models with a radial metallicity gradient, the vertical mixing of stars caused by the bar produces too shallow a vertical metallicity gradient. MartinezValpuesta \& Gerhard (2013) subsequently showed that a significantly steeper radial metallicity gradient, of $\approx-0.4$ $\mathrm{dex} / \mathrm{kpc}$, can indeed be transformed into a vertical gradient similar to the observed. A significant failure of this hypothesis is that the resulting bimodal distance distribution in the bulge (the 'X-shape') is more prominent in metal-poor populations, whereas in the MW the $\mathrm{X}$-shape is more prominent in metal-rich populations (Ness et al. 2012; Uttenthaler et al. 2012; Rojas-Arriagada et al. 2014). An in-situ picture of the vertical gradient relying on the superposition of a metal-rich thin disc and a metal-poor thick disc (Bekki \& Tsujimoto 2011; Di Matteo 2016), produces a gradient via the transition from one population to another. An alternative approach to producing the observed metallicity gradient was presented by Debattista et al. (2017) who found, in their simulation with self-consistent star formation and chemistry, a vertical metallicity gradient that resulted from the ability of older, metal-poor stars to reach larger heights during the buckling instability since they have larger radial random motion, compared to slightly ( $\sim 1 \mathrm{Gyr}$ ) younger stars. The resulting X-shape is better traced by the metal-rich populations, as observed. They also showed that as a consequence the metallicity distribution is more peanut-shaped than the density itself (see also Athanassoula et al. 2017; Buck et al. 2018; Debattista et al. 2019). Such a metallicity distribution has been confirmed in the edge-on galaxy NGC 4710, which hosts a B/P bulge (Gonzalez et al. 2017).

These trends help constrain models of the evolution of $\mathrm{B} / \mathrm{P}$ bulges. We found that side-on maps of $\mathrm{J}_{z, 0}$ and $\mathrm{J}_{\mathrm{R}, 0}$ are pinched and that the bimodal populations at fixed heights are less separated with increasing action. These are trends reminiscent of what is observed in the MW's bulge. If metallicity depends on $\mathrm{J}_{z, 0}$ then a bimodality forms but the formation of the bar and $\mathrm{B} / \mathrm{P}$ bulge substantially weakens the vertical gradient (Bekki \& Tsujimoto 2011). In this case we find that the initial vertical gradient is substantially weakened. Instead metallicity based on $\mathrm{J}_{\mathrm{R}, 0}$ will produce a strong and monotonic vertical gradient, as observed in the MW. This is an efficient way to generate vertical gradients from a system in which the in-plane random motions are a function of age by the time the bar forms. However we argue that $\mathrm{J}_{\mathrm{R}, 0}$ by itself is not sufficient to properly assign metallicities to particles. We show that assigning metallicities using all three actions results in a model that has the right X-shape metallicity variation and a vertical metallicity gradient not very different from the MW's. 


\subsection{Outlook for action-based metallicity assignment}

At this time, pure $N$-body (collisionless) simulations with $10^{8}$ particles do not represent significant computational difficulties (e.g. Sellwood 2012; D'Onghia et al. 2013). The collisionless cosmological simulation of Potter et al. (2017) employed $2 \times 10^{12}$ particles, which required only a modest 350,000 node hours to complete. On the other hand, hydrodynamical simulations with this number of particles constitute a huge computational effort. For instance, the Illustris TNG100 simulation, one of the IllustrisTNG flagship runs with $1.2 \times 10^{10}$ resolution elements, required $\sim 18 \mathrm{M}$ cpu hours to complete (Nelson et al. 2018; Marinacci et al. 2018; Pillepich et al. 2018; Naiman et al. 2018; Springel et al. 2018), with other runs in the IllustrisTNG suite requiring longer for varying resolutions. While these are extreme examples (and also differ in their resolutions), they demonstrate the very different cost of running pure $N$-body versus hydrodynamical simulations. Action-based stellarpopulation modelling therefore holds great promise for helping to understand the distribution of stellar populations within $\mathrm{B} / \mathrm{P}$ bulges by permitting rapid studies in parameter space using $N$-body simulations.

We have presented a simple experiment in assigning chemistry using the three actions. The model exhibits many of the trends of real B/P bulges. Since our simulations are pure $N$-body, with no star formation, their evolution covers only stellar populations extant at the time of bar formation. Because the bar grows by trapping disc particles, stars younger than the bar are not represented in the simulations. However, since stars forming after the bar do not substantially participate in the $\mathrm{B} / \mathrm{P}$ bulge (Debattista et al. 2017), this omission does not seriously affect our results on the stellar populations of the $\mathrm{B} / \mathrm{P}$ bulge itself. On the other hand, the stellar populations of the disc, where star formation continues even after the bar forms, cannot reliably be captured by our action-based metallicity assignment. Nevertheless, we conclude that within the main bulk of the $\mathrm{B} / \mathrm{P}$ bulge itself, the action-based metallicity assignment we have developed is a very useful tool for studying the stellar distribution. The method can be adapted in a variety of ways, including replacing the donor with an analytic prescription for the metallicity-action relation, including more moments in the metallicity distribution function of the donor, or mapping based on machine learning techniques.

\subsection{Summary}

We have computed the actions of star particles in the initial conditions of a suite of pure $N$-body simulations. We followed the particles to the point where a $\mathrm{B} / \mathrm{P}$ bulge forms, and examined how the initial actions were distributed in the final $\mathrm{B} / \mathrm{P}$ bulge. We also used a star-forming simulation, matched in action space, to assign metallicity to the particles of a pure $N$-body simulation. Our main results are:

Both the radial action, $\mathrm{J}_{\mathrm{R}, 0}$, and the vertical action, $\mathrm{J}_{\mathrm{z}, 0}$, separate stellar populations such that those with lower actions support a stronger, more elongated bar while those with higher actions host a weaker bar. The bar strength has a greater dynamic range when stellar populations are separated by $\mathrm{J}_{\mathrm{R}, 0}$ than by $\mathrm{J}_{\mathrm{z}, 0}$ (see Section 3.1).
- The central vertical profile of $\left\langle\mathrm{J}_{z, 0}\right\rangle$ is substantially flattened by the formation of the $\mathrm{B} / \mathrm{P}$ bulge. A vertical gradient develops in $\left\langle\mathrm{J}_{\phi, 0}\right\rangle$ (and also in $\left\langle R_{0}\right\rangle$, the mean radius of stars at $t=0)$, which reaches a peak and then declines at larger heights. The initially rather flat profile of $\left\langle\mathrm{J}_{\mathrm{R}, 0}\right\rangle$ is transformed into a monotonically rising profile by the $\mathrm{B} / \mathrm{P}$ bulge formation (see Sections 3.2 and 3.3).

- Stellar populations separated by either $\mathrm{J}_{\mathrm{R}, 0}$ or $\mathrm{J}_{\mathrm{z}, 0}$ display an increasing separation of the peaks of the bimodality with increasing height. The separation is stronger for the populations with lower $\mathrm{J}_{\mathrm{R}, 0}$ and lower $\mathrm{J}_{z, 0}$, and higher $\mathrm{J}_{\phi, 0}$. This different trend with $\mathrm{J}_{\phi, 0}$ would imply a greater separation in the X-shape in metal-poor populations if metallicity depended on angular momentum, or radius (see Section 3.4).

- The thickness at the end of the simulation, and the overall vertical thickening, increases monotonically with $J_{R, 0}$. This is the characteristic of kinematic fractionation described by Debattista et al. (2017). In contrast, the thickening is peaked at intermediate $\mathrm{J}_{\mathrm{z}, 0}$, decreasing to larger values and becoming negative (i.e. the populations become thinner) for the highest $\mathrm{J}_{\mathrm{z}, 0}$. Conyersely, the populations with the largest $\mathrm{J}_{\mathrm{R}, 0}$ radially cook (see Section 3.5).

- Only models with a narrow range of $\mathrm{J}_{\mathrm{R}, 0}$ diverge from these trends, and exhibiting a weak dependence of the bar strength and X-shape on the actions. These contrary behaviours serve to further illustrate the importance of the radial action in the development of the trends observed in stellar populations observed in the MW (see Section 4).

- We demonstrate a simple action-based mapping for setting the metallicity of star particles in a pure $N$-body simulation based on their actions using the metallicity of particles in a star-forming simulation. The resulting metallicity map of the galaxy seen edge-on with the bar seen side-on is pinched, matching observations in real galaxies and hydrodynamical models of barred galaxies (see Section 5.2.2).

In future papers we will present further analysis of these models and employ the action-based metallicity tagging to develop models of the MW's bulge chemistry.

\section{Acknowledgements.}

V.P.D. and L.B.S. are supported by Science and Technology Facilities Council Consolidated grant \# ST/R000786/1. D.J.L. was supported for part of this project by a UCLan UURIP internship. The simulations were run at the DiRAC Shared Memory Processing system at the University of Cambridge, operated by the COSMOS Project at the Department of Applied Mathematics and Theoretical Physics on behalf of the STFC DiRAC HPC Facility (www.dirac.ac.uk). This equipment was funded by BIS National E-infrastructure capital grant ST/J005673/1, STFC capital grant ST/H008586/1 and STFC DiRAC Operations grant ST/K00333X/1. DiRAC is part of the National EInfrastructure. We thank Larry Widrow and Nathan Deg for their assistance with GALACTICS and Eugene Vasiliev for his help with AGAMA. We thank the anonymous referee for comments that helped improve the paper. V.P.D. thanks Bruno Debattista for his help recovering the data for this paper after a laptop failure at the start of COVID-19 lockdown.

\section{Data availability}

The simulation data underlying this article will be shared on reasonable request to V.P.D (vpdebattista@gmail.com). 


\section{REFERENCES}

Abbott, C. G., Valluri, M., Shen, J., \& Debattista, V. P. 2017, MNRAS, 470, 1526

Araki, S. 1985, Ph.D. Thesis, MIT

Athanassoula, E. 2005, MNRAS, 358, 1477

Athanassoula, E., Rodionov, S. A., \& Prantzos, N. 2017, MNRAS, 467, L46

Barbanis, B., \& Woltjer, L. 1967, ApJ, 150, 461

Bekki, K., \& Tsujimoto, T. 2011, MNRAS, 416, L60

Berentzen, I., Heller, C. H., Shlosman, I., \& Fricke, K. J. 1998, MNRAS, 300, 49

Binney, J. 2012, MNRAS, 426, 1324

Binney, J., \& Lacey, C. 1988, MNRAS, 230, 597

Binney, J., \& Tremaine, S. 2008, Galactic Dynamics: Second Edition (Galactic Dynamics: Second Edition, by James Binney and Scott Tremaine. ISBN 978-0-69113026-2 (HB). Published by Princeton University Press, Princeton, NJ USA, 2008.)

Buck, T., Ness, M. K., Macciò, A. V., Obreja, A., \& Dutton, A. A. 2018, ApJ, 861, 88

Bureau, M., \& Athanassoula, E. 2005, ApJ, 626, 159

Carlberg, R. G., \& Sellwood, J. A. 1985, ApJ, 292, 79

Cole, D. R., \& Binney, J. 2017, MNRAS, 465, 798

Collier, A. 2020, MNRAS, 492, 2241

Combes, F., Debbasch, F., Friedli, D., \& Pfenniger, D. 1990, A\&A, 233, 82

Combes, F., \& Sanders, R. H. 1981, A\&A, 96, 164

Daniel, K. J., \& Wyse, R. F. G. 2015, MNRAS, 447, 3576

Debattista, V. P., Carollo, C. M., Mayer, L., \& Moore, B. 2004, ApJ, 604, L93

Debattista, V. P., Carollo, C. M., Mayer, L., \& Moore, B. 2005, ApJ, 628, 678

Debattista, V. P., Gonzalez, O. A., Sanderson, R. E., ElBadry, K., Garrison-Kimmel, S., Wetzel, A., FaucherGiguère, C.-A., \& Hopkins, P. F. 2019, MNRAS, 485, 5073

Debattista, V. P., Mayer, L., Carollo, C. M., Moore, B., Wadsley, J., \& Quinn, T. 2006, ApJ, 645, 209

Debattista, V. P., Ness, M., Gonzalez, O. A., Freeman, K., Zoccali, M., \& Minniti, D. 2017, MNRAS, 469, 1587

Di Matteo, P. 2016, PASA, 33, 027

Di Matteo, P., Fragkoudi, F., Khoperskov, S., Ciambur, B., Haywood, M., Combes, F., \& Gómez, A. 2019, A\&A, 628, A11

Di Matteo, P., et al. 2015, A\&A, 577, A1

Di Matteo, P., et al. 2014, A\&A, 567, A122

D'Onghia, E., Vogelsberger, M., \& Hernquist, L. 2013, ApJ, 766,34

Donohoe-Keyes, C. E., Martig, M., James, P. A., \& Kraljic, K. 2019, MNRAS, 489, 4992

Erwin, P., \& Debattista, V.P. 2016, ApJ, 825, L30

Erwin, P., \& Debattista, V. P. 2017, MNRAS, 468, 2058

Fouvry, J.-B., \& Bar-Or, B. 2018, MNRAS, 481, 4566

Fragkoudi, F., Di Matteo, P., Haywood, M., Gómez, A., Combes, F., Katz, D., \& Semelin, B. 2017, A\&A, 606, A47

Fragkoudi, F., Di Matteo, P., Haywood, M., Schultheis, M., Khoperskov, S., Gómez, A., \& Combes, F. 2018, A\&A, 616, A180

Gonzalez, O. A., Debattista, V. P., Ness, M., Erwin, P., \& Gadotti, D. A. 2017, MNRAS, 466, L93

Gonzalez, O. A., et al. 2011, A\&A, 530, A54
Gonzalez, O. A., Rejkuba, M., Zoccali, M., Valent, E., Minniti, D., \& Tobar, R. 2013, A\&A, 552, A110

Hayden, M. R., et al. 2015, ApJ, 808, 132

Heyvaerts, J. 2010, MNRAS, 407, 355

Heyvaerts, J., Fouvry, J.-B., Chavanis, P.-H., \& Pichon, C. 2017, MNRAS, 469, 4193

Icke, V. 1982, ApJ, 254, 517

James, P. A., \& Percival, S. M. 2016, MNRAS, 457, 917

James, P. A., \& Percival, S. M. 2018, MNRAS, 474, 3101

Johnson, C. I., Rich, R. M., Fulbright, J. P., Valenti, E., \& McWilliam, A. 2011, ApJ, 732, 108

Johnson, C. I., Rich, R. M., Kobayashi, C., Kunder, A., Pilachowski, C. A., Koch, A., \& de Propris, R. 2013, ApJ, 765,157

Kruk, S. J., Erwin, P., Debattista, V. P., \& Lintott, C. 2019, MNRAS, 490, 4721

Kuijken, K., \& Dubinski, J. 1995, MNRAS, 277, 1341

Lacey, C. G. 1984, MNRAS, 208, 687

Li, Z.-Y., Ho, L. C., \& Barth, A. J. 2017, ApJ, 845, 87

Li, Z.-Y., \& Shen, J. 2012, ApJ, 757, L7

Li, Z.-Y., \& Shen, J. 2015, ApJ, 815, L20

Loebman, S. R., Debattista, V. P., Nidever, D. L., Hayden, M. R., Holtzman, J. A., Clarke, A. J., Roškar, R., \& Valluri, M. 2016, ApJ, 818, L6

Loebman, S. R., Roškar, R., Debattista, V. P., Ivezić, Ž., Quinn, T. R., \& Wadsley, J. 2011, ApJ, 737, 8

Łokas, E. L. 2019, A\&A, 629, A52

Lynden-Bell, D, \& Kalnajs, A. J. 1972, MNRAS, 157, 1

Marinacci, F., et al. 2018, MNRAS, 480, 5113

Martinez-Valpuesta, 1., \& Gerhard, O. 2011, ApJ, 734, L20

Martinez-Valpuesta, I., \& Gerhard, O. 2013, ApJ, 766, L3

Martinez-Valpúesta, I., \& Shlosman, I. 2004, ApJ, 613, L29

Martinez-Valpuesta, I., Shlosman, I., \& Heller, C. 2006, ApJ, 637, 214

Merritt, D., \& Sellwood, J. A. 1994, ApJ, 425, 551

Molaeinezhad, A., Falcón-Barroso, J., Martínez-Valpuesta, I., Khosroshahi, H. G., Vazdekis, A., La Barbera, F., Peletier, R. F., \& Balcells, M. 2017, MNRAS, 467, 353

Naiman, J. P., et al. 2018, MNRAS, 477, 1206

Navarro, J. F., Frenk, C. S., \& White, S. D. M. 1996, ApJ, 462,563

Nelson, D., et al. 2018, MNRAS, 475, 624

Nelson, R. W., \& Tremaine, S. 1999, MNRAS, 306, 1

Ness, M., et al. 2013, MNRAS, 430, 836

Ness, M., et al. 2012, ApJ, 756, 22

O'Neill, J. K., \& Dubinski, J. 2003, MNRAS, 346, 251

Pfenniger, D., \& Friedli, D. 1991, A\&A, 252, 75

Pillepich, A., et al. 2018, MNRAS, 475, 648

Portaluri, E., et al. 2017, MNRAS, 467, 1008

Potter, D., Stadel, J., \& Teyssier, R. 2017, Computational Astrophysics and Cosmology, 4, 2

Raha, N., Sellwood, J. A., James, R. A., \& Kahn, F. D. 1991, Nature, 352, 411

Rojas-Arriagada, A., et al. 2014, A\&A, 569, A103

Roškar, R., Debattista, V. P., Brooks, A. M., Quinn, T. R., Brook, C. B., Governato, F., Dalcanton, J. J., \& Wadsley, J. 2010, MNRAS, 408, 783

Roškar, R., Debattista, V. P., Quinn, T. R., \& Wadsley, J. 2012, MNRAS, 426, 2089

Roškar, R., Debattista, V. P., Stinson, G. S., Quinn, T. R., Kaufmann, T., \& Wadsley, J. 2008, ApJ, 675, L65 Saha, K., \& Gerhard, O. 2013, MNRAS, 430, 2039 
Saha, K., Pfenniger, D., \& Taam, R. E. 2013, ApJ, 764, 123

Sellwood, J. A. 1981, A\&A, 99, 362

Sellwood, J. A. 2012, ApJ, 751, 44

Sellwood, J. A. 2013, ApJ, 769, L24

Sellwood, J. A. 2015, MNRAS, 453, 2919

Sellwood, J. A., \& Athanassoula, E. 1986, MNRAS, 221, 195

Sellwood, J. A., \& Binney, J. J. 2002, MNRAS, 336, 785

Shen, J., Rich, R. M., Kormendy, J., Howard, C. D., De Propris, R., \& Kunder, A. 2010, ApJ, 720, L72

Smirnov, A. A., \& Sotnikova, N. Y. 2019, MNRAS, 485, 1900

Solway, M., Sellwood, J. A., \& Schönrich, R. 2012, MNRAS, 422, 1363

Spitzer, L., Jr., \& Schwarzschild, M. 1951, ApJ, 114, 385

Spitzer, L., Jr., \& Schwarzschild, M. 1953, ApJ, 118, 106

Springel, V., et al. 2018, MNRAS, 475, 676

Stadel, J. G. 2001, Ph.D. Thesis, University of Washington

Toomre, A. 1981, in Structure and Evolution of Normal Galaxies, ed. S. M., Fall \& D. Lynden-Bell (Cambridge: Cambridge University Press), 111

Uttenthaler, S., Schultheis, M., Nataf, D. M., Robin, A. C., Lebzelter, T., \& Chen, B. 2012, A\&A, 546, A57

Valluri, M., Shen, J., Abbott, C., \& Debattista, V. P. 2016 , ApJ, 818, 141

Vasiliev, E. 2019, MNRAS, 482, 1525

Vera-Ciro, C., \& D’Onghia, E. 2016, ApJ, 824, 39

Villumsen, J. V. 1985, ApJ, 290, 75

Wegg, C., Gerhard, O., \& Portail, M. 2015, MNRAS, 450, 4050

Widrow, L. M., \& Dubinski, J. 2005, ApJ, 631, 838

Widrow, L. M., Pym, B., \& Dubinski, J. 2008, ApJ, 679, 1239

Wielen, R. 1977, A\&A, 60, 263

Zoccali, M., Hill, V., Lecureur, A., Barbuy, B., Renzini, A.,

Minniti, D., Gómez, A., \& Ortolani, S. 2008, A\&A, 486, 177

Zoccali, M., et al. 2017, A\&A, 599, A12 